\title{
Constraining cosmology with the cosmic microwave and infrared backgrounds correlation
}

\author{
A. Maniyar ${ }^{1}$, G. Lagache $^{1}$, M. Béthermin ${ }^{1}$, and S. Ilić ${ }^{2}$ \\ 1 Aix Marseille Univ, CNRS, CNES, LAM, Marseille, France \\ e-mail: abhishek.maniyar@lam. fr \\ 2 CEICO, Institute of Physics of the Czech Academy of Sciences, Na Slovance 2, Praha 8, Czech Republic
}

Received 3 July 2018 / Accepted 12 September 2018

\begin{abstract}
We explore the use of the cosmic infrared background (CIB) as a tracer of the large scale structures for cross-correlating with the cosmic microwave background (CMB) and exploit the integrated Sachs-Wolfe (ISW) effect. We used an improved linear CIB model from our previous work and derived the theoretical CIB $\times$ ISW cross-correlation for different Planck HFI frequencies (217, 353, 545 and $857 \mathrm{GHz})$ and IRAS $(3000 \mathrm{GHz})$. As expected, we predict a positive cross-correlation between the CIB and the CMB whose amplitude decreases rapidly at small scales. We perform a signal-to-noise ratio $(\mathrm{S} / \mathrm{N})$ analysis of the predicted cross-correlation. In the ideal case when the cross-correlation is obtained over $70 \%$ (40\%) of the sky without residual contaminants (e.g. galactic dust) in maps, the $\mathrm{S} / \mathrm{N}$ ranges from 4.2 to 5.6 (3.2 to 4.3); the highest $\mathrm{S} / \mathrm{N}$ comes from $857 \mathrm{GHz}$. A Fisher matrix analysis shows that an ISW signal detected with a $\mathrm{S} / \mathrm{N}$ this high on the $40 \%$ sky can considerably improve the constraints on the cosmological parameters; constraints on the equation of state of the dark energy especially are improved by $80 \%$. We then performed a more realistic analysis considering the effect of residual galactic dust contamination in CIB maps. We calculated the dust power spectra for different frequencies and sky fractions that dominate the CIB power spectra at the lower multipoles we are interested in. Considering a conservative $10 \%$ residual level of galactic dust in the CIB power spectra, we observe that the S/N drops drastically, which makes it very challenging to detect the ISW. To determine the capability of current maps to detect the ISW effect through this method, we measured the cross-correlation of the CIB and the CMB Planck maps on the so-called GASS field, which covers an area of $\sim 11 \%$ in the southern hemisphere. We find that with such a small sky fraction and the dust residuals in the CIB maps, we do not detect any ISW signal, and the measured cross-correlation is consistent with zero. To avoid degrading the $\mathrm{S} / \mathrm{N}$ for the ISW measurement by more than $10 \%$ on the $40 \%$ sky, we find that the dust needs to be cleaned up to the $0.01 \%$ level on the power spectrum.
\end{abstract}

Key words. infrared: diffuse background - cosmic background radiation - large-scale structure of Universe cosmological parameters - cosmology: observations - methods: statistical

\section{Introduction}

At the end of the last century, Riess et al. (1998) and Perlmutter et al. (1999) discovered that the expansion of the Universe is accelerating. Since then, a great wealth of theories have been put forward to explain this acceleration. The leading theory attributes it to so-called "dark energy", an unknown component constituting around $70 \%$ of the total energy budget of the Universe according to our latest estimates (Planck Collaboration XIII 2016). The $\Lambda$ cold dark matter (CDM) model, also called the concordance model, is favoured most by the data in the context of a Bayesian model comparison (Planck Collaboration XIII 2016). Currently, great effort is dedicated to constraining the parameters of our cosmological model better. Various cosmological probes of the distance scales (all the standard rulers and candles, i.e. baryonic acoustic oscillations Eisenstein \& Hu 1998, supernovae Ia Betoule et al. 2014, cepheids Riess et al. 2016) and probes of the growth of structures (weak lensing Lewis \& Challinor 2006, galaxy clustering Tinker et al. 2012, redshift space distortions Scoccimarro 2004) are being used to study and test cosmological models such as the $\Lambda \mathrm{CDM}$ and possible deviations from it.

One of these is the integrated Sachs-Wolfe (ISW) effect. This is another potential powerful probe of dark energy. It is the low-redshift integrated counterpart of the original Sachs-Wolfe effect (Sachs \& Wolfe 1967) that occurs at the surface of the last scattering and leaves its imprints on the cosmic microwave background (CMB) anisotropies. This effect is caused by the gravitational redshifting of the photons when they exit the potential wells at the last scattering surface. The ISW effect, on the other hand, occurs in a universe that is not dominated by matter. As dark energy starts to dominate the dynamics of the Universe at late times, it causes a decay of the gravitational potential wells and hills on large scales. As a result, the CMB photons travelling across them have a net gain or loss of energy for potential wells and hills, respectively. The ISW effect is the dominant contribution to the $\mathrm{CMB}$ power spectrum at large angular scales and has a very small amplitude. Unfortunately, at these scales, the statistical noise that is due to cosmic variance in the CMB power spectrum is of the same order of magnitude as the signal, which makes the detection of the ISW effect in the CMB alone extremely challenging.

However, we know that the matter in the Universe shapes the underlying gravitational potentials that are responsible for the ISW effect. Therefore, a non-zero spatial correlation between the CMB anisotropies and tracers of the matter distribution is expected, and can be exploited to extract the ISW signal (e.g. Boughn et al. 1998; Boughn \& Crittenden 
2002). Galaxy surveys (which are proxies for large-scale structures) are an obvious choice for this purpose. Attempts to detect the ISW signal have been made with various surveys, such as Wide-field Infrared Survey Explorer (WISE; Shajib \& Wright 2016; Ferraro et al. 2015), Sloan Digital Sky Survey (SDSS; Cabré et al. 2006; Giannantonio et al. 2006; Hernández-Monteagudo et al. 2014), NRAO VLA Sky Survey (NVSS; Raccanelli et al. 2008, Pietrobon et al. 2006), and Two Micron All-Sky Survey (2MASS; Francis \& Peacock 2010). Joint analyses combining different data sets have also been performed (Giannantonio et al. 2008; Planck Collaboration XXI 2016; Stölzner et al. 2018). Alternative approaches have also been explored in the literature, such as stacking CMB patches at the location of superstructures that are expected to yield the strongest ISW signal (Ilić et al. 2013) and exploiting the cross-correlation between the ISW and thermal SunyaevZel'dovich effects, which are both present in the CMB and take place in the same gravitational wells (Taburet et al. 2011; Creque-Sarbinowski et al. 2016). The significance of the ISW signals detected in all these studies ranges from $1 \sigma$ to $5 \sigma$, and the main limiting factor is the lack of sky coverage and/or the redshift depth of the surveys. These are both crucial factors in the detection of the ISW effect because the largest structures contribute most and thus require the largest possible survey volume.

We here consider the cosmic infrared background (CIB) as a tracer of the large-scale structures to probe the ISW effect. The CIB is the weighted integral of the dust heated by the young UV-bright stars within the galaxies through cosmic time. It was first detected by Puget et al. (1996). As it traces the star formation history of the Universe, it spans a wide redshift range of $0<z<6$. Therefore, the CIB is an exceptional tool for tracing the overall distribution of the galaxies at these high redshifts (Knox et al. 2001). Lagache \& Puget (2000) and Matsuhara et al. (2000) first detected and discussed the anisotropies in the CIB that are due to unresolved extragalactic sources. Correlated anisotropies in the CIB were discovered by Spitzer (Lagache et al. 2007) and were accurately measured by Planck and Herschel (Planck Collaboration XXX 2014; Viero et al. 2013b). These anisotropies trace the large-scale distribution of the galaxy density field and consequently, the underlying distribution of the dark matter haloes that host these galaxies, up to a bias factor. This means that the CIB can be used as a tracer of the large-scale structures (e.g. Hanson et al. 2013).

As the CIB traces the evolution of the large-scale structures over a wide range of redshifts, it is a prime choice of tracer for detecting the ISW effect. Ilić et al. (2011) showed that the $\mathrm{CIB} \times \mathrm{ISW}$ cross-correlation can provide us with the highest signal-to-noise ratio $(\mathrm{S} / \mathrm{N})$ for a single tracer to detect the ISW signal. Since then, our understanding and modelling of the CIB anisotropies has improved (e.g. Béthermin et al. 2013; Viero et al. 2013a; Wu et al. 2018), and we have gained new insights on their contaminants or foregrounds and thus on the intricacies of extracting the CIB signal (Planck Collaboration XXX 2014). We thus revisit the prediction for the cross-correlation between the CIB and the CMB. Maniyar et al. (2018) simultaneously considered the CIB autoand cross-power spectra as well as the cross-correlation of the $\mathrm{CIB} \times \mathrm{CMB}$ lensing measurements to constrain an updated model for the CIB anisotropies. Here we use this model and predict the expected $\mathrm{S} / \mathrm{N}$ for the ISW effect obtained through the $\mathrm{CIB} \times \mathrm{CMB}$ cross-correlation. We then perform a Fisher matrix analysis to predict the constraints on the cosmological parameters obtained from a combination of the CIB $\times$ ISW information with $\mathrm{CMB}$, $\mathrm{CIB}$, and $\mathrm{CIB} \times \mathrm{CMB}$ lensing data. Unfortunately, current $\mathrm{CIB}$ maps are limited by the dust contamination, especially at lower angular multipoles, where the ISW signal is expected to be strongest. Therefore we perform our analysis in two scenarios: one for the ideal situation, in which dust-free CIB spectra are extracted over the full or partial sky, and the second case for CIB maps that are contaminated by residual galactic dust emission.

This paper is structured as follows. We begin in Sect. 2 by introducing the modelling of the various cosmological probes considered in our analysis: our model for the CIB power spectrum, the $\mathrm{CIB} \times \mathrm{CMB}$ lensing correlation, and the $\mathrm{CIB} \times \mathrm{ISW}$ correlation. In Sect. 3 we provide the predictions for the ISW signal and its $\mathrm{S} / \mathrm{N}$ for different cases (maps with and without residual dust contamination). In Sect. 4 we explain the Fisher matrix analysis we used to constrain the cosmological parameters and provide the results for the $\Lambda \mathrm{CDM}$ and $w \mathrm{CDM}$ models. Section 5 details the measurement of the cross-correlation using the available CIB and CMB Planck maps over a small portion of the sky and the corresponding results. Conclusions are provided in Sect. 6. Throughout our analysis, we use the Planck 2015 bestfit cosmology (Planck Collaboration XIII 2016) with adiabatic scalar perturbations and a nearly scale-invariant initial power spectrum.

\section{Cosmological observables and their modelling}

\subsection{CIB power spectrum}

We aim at exploiting the cross-correlation of the CIB with the CMB through the ISW effect, which is mostly significant on large angular scales. At these scales, the clustering signal is dominated by the correlation between the dark matter halos, and it is safe to ignore the non-linear effects. Thus, a linear model for calculating the CIB anisotropies is good enough for our study. The CIB power spectrum is defined as

$C_{l}^{v \times v^{\prime}} \times \delta_{l l^{\prime}} \delta_{m m^{\prime}}=\left\langle\delta I_{l m}^{v} \delta I_{l^{\prime} m^{\prime}}^{v^{\prime}}\right\rangle$.

The CIB anisotropies for a given frequency $v$ at redshift $z$ and in a given direction $\hat{\mathbf{n}}$ are

$\delta I_{\mathrm{CIB}}(\hat{\mathbf{n}}, v, z)=\int \mathrm{d} z \frac{\mathrm{d} \chi}{\mathrm{d} z} a(z) \delta j(\hat{\mathbf{n}}, v, z)$,

where $I_{\mathrm{CIB}}$ is the CIB mean level, $\chi(z)$ is the comoving distance to redshift $z$ and $a=1 /(1+z)$ is the scale factor, and $j$ is the comoving emissivity of the CIB galaxies.

We used the CIB model presented in Maniyar et al. (2018). In order to fit for the model parameters, only CIB data in the range $145<\ell<592$ were used, where the linear model is a good approximation and the Limber approximation is accurate enough and simplifies the calculation. The linear CIB anisotropy power spectrum with the Limber approximation is then given as

$C_{l}^{v \times v^{\prime}}=\int \frac{\mathrm{d} z}{\chi^{2}} \frac{\mathrm{d} \chi}{\mathrm{d} z} a^{2} b_{\text {eff }}^{2} \bar{j}(v, z) \bar{j}\left(v^{\prime}, z\right) P_{\text {lin }}(k=l / \chi, z)$.

where $P_{\text {lin }}(k, z)$ is the linear theory dark matter power spectrum, which has been generated using the publicly available Boltzmann code CAMB (Lewis \& Bridle 2002; Lewis 2014) at the required redshifts. $b_{\text {eff }}$ is the effective bias factor for the CIB galaxies at a given redshift. This is the mean bias of dark matter halos hosting dusty galaxies that contribute to the CIB at a given redshift weighted by their contribution to the emissivities (Planck Collaboration XXX 2014; Maniyar et al. 2018). It implies that more massive halos are more clustered and that 
their host galaxies emit more far-infrared emission. We used a parametric form for the evolution of the effective bias with the redshift

$b_{\mathrm{eff}}(z)=b_{0}+b_{1} z+b_{2} z^{2}$.

The bias only depends on the redshift and is scale independent. This is a good approximation at the scales where we model the CIB.

$\bar{j}$ is the comoving emissivity (in $\mathrm{Jy} \mathrm{Mpc}^{-1}$ ), which is derived using the star formation rate (SFR) density $\rho_{\mathrm{SFR}}$ $\left(M_{\odot} \mathrm{yr}^{-1} \mathrm{Mpc}^{-3}\right)$ following

$\bar{j}(v, z)=\frac{\rho_{\mathrm{SFR}}(z)(1+z) S_{v, \mathrm{eff}}(z) \chi^{2}(z)}{K}$,

where $K$ is the Kennicutt constant (Kennicutt 1998). We used a Kennicutt constant corresponding to a Chabrier initial mass function $\left(\mathrm{SFR} / L_{\mathrm{IR}}=1.0 \times 10^{-10} M_{\odot} \mathrm{yr}^{-1}\right) . S_{v, \mathrm{eff}}(z)\left(\right.$ in Jy $\left.L_{\odot}^{-1}\right)$ is the average spectral energy distribution (SED) of all the galaxies at a given redshift weighted by their contribution to the CIB. As described in Maniyar et al. (2018), they were computed using the method presented in Béthermin et al. (2013), but assuming the new updated SEDs that were calibrated with Herschel data, as presented in Béthermin et al. (2015, 2017). The parametric form of the cosmic SFR density proposed by Madau \& Dickinson (2014) is used to describe $\rho_{\mathrm{SFR}}$ and is given by

$\rho_{\mathrm{SFR}}(z)=\alpha \frac{(1+z)^{\beta}}{1+[(1+z) / \gamma]^{\delta}} M_{\odot} \mathrm{yr}^{-1} \mathrm{Mpc}^{-3}$,

where $\alpha, \beta, \gamma$, and $\delta$ are parameters of the CIB model.

\section{2. $C I B \times C M B$ lensing}

As mentioned earlier, the $\mathrm{CIB} \times \mathrm{CMB}$ lensing cross-correlation is another ingredient of our study, which we used to further constrain the parameters of our CIB model and the cosmological parameters. The CMB photons that propagate freely from the last scattering surface towards us are gravitationally deflected by the large-scale distribution of the matter in the Universe. The gravitational lensing leaves imprints on the CMB temperature and polarisation anisotropies. A map of the lensing potential along the line of sight can be reconstructed using these imprints (Okamoto \& Hu 2003). Primary sources for this CMB lensing potential are the dark matter halos that are located between us and the last scattering surface (Lewis \& Challinor 2006), and a strong correlation between the CIB anisotropies and a lensing-derived projected mass map has been shown to be expected (e.g. Song et al. 2003) and indeed measured (Planck Collaboration XVIII 2014) with a high S/N.

We calculated the cross-correlation between the CIB and the $\mathrm{CMB}$ lensing potential, which is given by

$C_{l}^{v \phi}=\int b_{\mathrm{eff}} \bar{j}(v, z) \frac{3}{l^{2}} \Omega_{\mathrm{m}} H_{0}^{2}\left(\frac{\chi_{*}-\chi}{\chi_{*} \chi}\right) P_{\operatorname{lin}}(k=l / \chi, z) \mathrm{d} \chi$,

where $\chi_{*}$ is the comoving distance to the CMB last scattering surface, $\Omega_{\mathrm{m}}$ is the matter density parameter, and $H_{0}$ is the value of the Hubble parameter today. From this equation, it is seen that $C_{l}^{v, \phi}$ is proportional to $b_{\text {eff }}$, whereas $C_{l}^{\mathrm{CIB}}$ is proportional to $b_{\mathrm{eff}}^{2}$. Using the $\mathrm{CIB} \times \mathrm{CMB}$ lensing potential measurement in the $\mathrm{CIB}$ model likelihood therefore also helps us resolve the degeneracy between the evolution of $b_{\mathrm{eff}}$ and $\rho_{\mathrm{SFR}}$ to some extent.

\section{3. $C I B \times I S W$ correlation}

The contribution of the ISW effect to the CMB temperature anisotropies is given by

$\delta T_{\mathrm{ISW}}(\hat{\mathbf{n}})=\int_{\eta_{r}}^{\eta_{0}} \mathrm{~d} \eta \mathrm{e}^{-\tau(\eta)}(\dot{\Phi}-\dot{\Psi})\left[\left(\eta_{0}-\eta\right) \hat{\mathbf{n}}, \eta\right]$.

It represents the integration of the time derivative of the $\Phi$ and $\Psi$ Newtonian gauge gravitational potentials (with Kodama \& Sasaki 1984 conventions) over the conformal time $\eta$, with $\eta_{\mathrm{r}}$ being the initial time deep in the radiation era, $\tau(\eta)$ is the optical depth, and dots denote differentiation with respect to the conformal time $\eta$.

The cross-correlation between the CIB and ISW effect for a given frequency is then

$C^{\mathrm{cr}}\left(\theta_{\hat{\mathbf{n}}_{1}, \hat{\mathbf{n}}_{2}, v}\right) \equiv\left\langle\delta T_{\mathrm{CIB}}\left(\hat{\mathbf{n}}_{1}, v\right) \delta T_{\mathrm{ISW}}\left(\hat{\mathbf{n}}_{2}\right)\right\rangle$,

which becomes, after decomposing in the Legendre series:

$C^{\mathrm{cr}}(\theta, v)=\sum_{l=2}^{\infty} \frac{2 l+1}{4 \pi} C_{l}^{\mathrm{cr}}(v) P_{l}(\cos (\theta))$,

where monopole and dipole are not included. The ISW effect is expected to be dominant on large angular scales $(\ell<100)$. At these scales, the Limber approximation is not accurate enough. Following the calculations of Garriga et al. (2004), we obtain the $\mathrm{CIB} \times \mathrm{ISW}$ cross power spectrum without Limber approximation as

$C_{l}^{\mathrm{cr}}(v)=4 \pi \frac{9}{25} \int \frac{\mathrm{d} k}{k} \Delta_{\mathcal{R}}^{2} T_{l}^{\mathrm{ISW}}(k) M_{l}(k, v)$,

where $\Delta_{\mathcal{R}}^{2}$ is the dimensionless primordial power spectrum related to the primordial power spectrum $\mathcal{P}_{\mathcal{R}} \equiv 2 \pi^{2} \Delta_{\mathcal{R}}^{2} / k^{3}$. The $M_{l}$ and $T_{l}^{\mathrm{ISW}}$ terms are

$M_{l}(k, v)=c_{\delta \Psi} \int_{\eta_{0}}^{\eta_{r}} \mathrm{~d} \eta j_{l}\left(k\left[\eta-\eta_{0}\right]\right) a(\eta) b_{\mathrm{eff}}(v) \bar{j}(v, \eta) \bar{\delta}(k, \eta)$

$T_{l}^{\mathrm{ISW}}(k)=\int_{\eta_{0}}^{\eta_{r}} \mathrm{~d} \eta \mathrm{e}^{-\tau(\eta)} j_{l}\left(k\left[\eta-\eta_{0}\right]\right)\left(c_{\Phi \Psi} \dot{\phi}-\dot{\psi}\right)$,

where $j_{l}$ are spherical Bessel functions, and $\bar{\delta}, \phi$ and $\psi$ are the time-dependent part of the dark matter density contrast $\delta$, and the Newtonian gauge gravitational potentials $\Phi$ and $\Psi$, respectively. The relation between the two coefficients $c_{\Psi \Phi}$ and $c_{\delta \Psi}$ for the adiabatic initial conditions is given as (Ma \& Bertschinger 1995)

$c_{\delta \Psi} \equiv \frac{\delta}{\Psi}=-\frac{3}{2}, \quad c_{\Phi \Psi} \equiv \frac{\Phi}{\Psi}=-\left(1+\frac{2}{5} R_{\nu}\right)$,

where $R_{v} \equiv \rho_{v} /\left(\rho_{v}+\rho_{\gamma}\right)$, with $\rho_{v}$ and $\rho_{\gamma}$ being the energy densities in relativistic neutrinos and photons, respectively. Later in this work, we compute the CIB spectrum at these low multipoles in order to derive the expected covariance of the $\mathrm{CIB} \times \mathrm{ISW}$ power spectrum (see details in Sect. 3). In order to be as consistent and rigorous as possible, in this context we use a different expression for the CIB power spectrum compared to Eq. (3), one that does not rely on the Limber approximation:

$C_{l}^{\mathrm{CIB}}(v)=4 \pi \frac{9}{25} \int \frac{\mathrm{d} k}{k} \Delta_{\mathcal{R}}^{2} M_{l}^{2}(k, v)$.

We modified the publicly available Boltzman code CAMB to compute our observables of interest: the CIB, CIB $\times I S W$, and $\mathrm{CIB} \times \mathrm{CMB}$ lensing power spectra, in addition to the standard CAMB outputs, that is, the $\mathrm{CMB}$ temperature and polarisation power spectra. 
Table 1. Parameter values of the CIB and cosmological models.

\begin{tabular}{cccccccc}
\hline \hline CIB model & $\alpha$ & $\beta$ & $\gamma$ & $\delta$ & $b_{0}$ & $b_{1}$ & $b_{2}$ \\
& 0.007 & 3.590 & 2.453 & 6.578 & 0.830 & 0.742 & 0.318 \\
\hline \multirow{2}{*}{ Cosmology } & $H_{0}$ & $w$ & $\Omega_{b} h^{2}$ & $\Omega_{c} h^{2}$ & $\tau$ & $n_{s}$ & $10^{9} A_{s}$ \\
& 67.47 & -1.019 & 0.022 & 0.119 & 0.062 & 0.965 & 2.128 \\
\hline
\end{tabular}

\section{Expected CIB $\times I S W$ cross-correlation and significance}

Using the formalism described in the previous section, we focus first on revisiting the predictions of Ilic et al. (2011) for the expected power spectrum and the $\mathrm{S} / \mathrm{N}$ of the $\mathrm{CIB} \times \mathrm{ISW}$ cross-correlation, using the improved and more tightly constrained CIB model of Maniyar et al. (2018) described earlier. Before proceeding further, a fiducial set of parameter needs to be defined in order to carry out our computations. For the sake of coherence, we followed Maniyar et al. (2018): as the CIB alone cannot constrain cosmological parameters, the authors first assumed a Planck fiducial background cosmology (Planck Collaboration XIII 2016), which we chose as our fiducial cosmological parameters throughout this work. The authors then derived the best-fit parameters of their CIB model using a combination of multi-frequency measurements of the CIB and $\mathrm{CIB} \times \mathrm{CMB}$ lensing power spectra from Planck and IRAS, as well as SFR density measurements and mean CIB intensity levels from external surveys. We use these best-fit CIB parameters as our fiducial values throughout this work. They are given in Table 1.

\section{1. $C I B \times I S W$ cross-correlation spectrum}

We computed the CIB $\times$ ISW cross power spectra for the Planck $217,353,545$, and $857 \mathrm{GHz}$ and IRAS $3000 \mathrm{GHz}$ frequency channels; these are the same frequencies as in the CIB datasets used by Maniyar et al. (2018). These power spectra are shown in Fig. 1. The amplitude of the cross-correlation is maximum around $20<\ell<50$ and falls off quickly at higher multipoles, as expected from the ISW effect. The amplitude of the power spectra increases with frequency. This is expected as the CIB probes galaxies at higher redshifts in low-frequency channels (and vice versa) and the main contribution to the ISW effect comes from low redshifts. This can be appreciated from the CIB anisotropy redshift distributions provided in Fig. 4 of Maniyar et al. (2018), which shows that the distribution peaks towards lower redshifts with increasingly higher frequencies. The amplitude of the signal is the highest for $857 \mathrm{GHz}$, which is slightly higher than the amplitude at $3000 \mathrm{GHz}$. The reason behind this is that the CIB has slightly more power at lower multipoles at $857 \mathrm{GHz}$ than at $3000 \mathrm{GHz}$. We did not consider the signal at higher multipoles $(\ell>100)$ as the non-linear counterpart to the ISW effect (the Rees-Sciama effect) starts to contribute significantly to the CMB anisotropies at these scales. The linear ISW effect still dominates near the peak of the signal, however (Seljak 1996).

\subsection{Signal-to-noise ratio}

After calculating the expected $\mathrm{CIB} \times \mathrm{ISW}$ cross-correlation signal, we determined the significance (detection level) of the signal. To do so, we performed an $\mathrm{S} / \mathrm{N}$ analysis. The cumulative $\mathrm{S} / \mathrm{N}$ for the ISW effect summed over all the multipoles between $2 \leq \ell \leq 100$ (where most of the signal resides, as shown in Fig. 1)

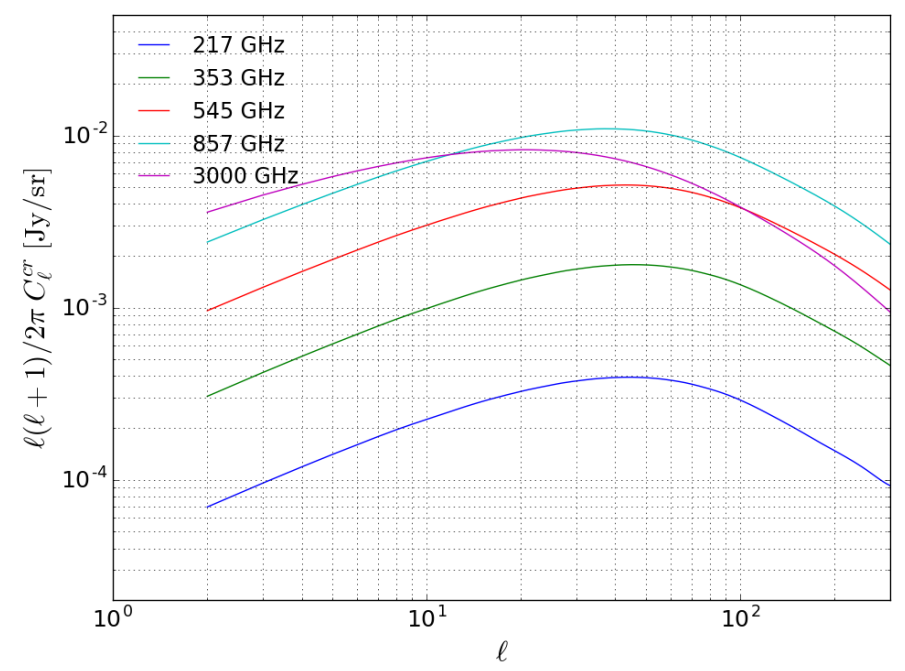

Fig. 1. CIB $\times I S W$ cross-correlation power spectra (in $\mathrm{Jy} \mathrm{sr}^{-1}$ ) for the five frequency channels.

for a given frequency $v$ can be written as

$\left[\frac{S}{N}\right]^{2}(v)=\sum_{\ell=2}^{\ell_{\max }}\left(\frac{S_{\ell}(v)}{N_{\ell}(v)}\right)^{2}$,

where the signal term $S_{\ell}$ in our case is the cross-correlation power spectrum:

$S_{\ell}(v) \equiv C_{\ell}^{\mathrm{cr}}(v)$,

and the noise term $N_{\ell}$ corresponds to the square root of its variance, the usual cosmic variance:

$N_{\ell}(v) \equiv \sqrt{\frac{\left[C_{\ell}^{\mathrm{cr}}(v)\right]^{2}+C_{\ell}^{\mathrm{CIB}}(v) C_{\ell}^{\mathrm{CMB}}}{2 \ell+1}}$.

For this analysis, we considered two cases that we detail below.

Ideal case. We assumed that the CIB and the CMB maps we used to calculate the cross-correlation were extracted over the full sky and are completely free of residual contaminations (we note that instrument noise is not relevant at the angular scales of the ISW effect). The only limiting factor is the cosmic variance, and we obtain the highest $\mathrm{S} / \mathrm{N}$ in this scenario. With these ideal assumptions, the $\mathrm{CIB} \times \mathrm{ISW}$ correlation reaches a significance level as high as $\approx 6.7 \sigma$. Detailed results are provided in Table 2 . Cumulative S/Ns for different Planck and IRAS frequencies are shown in Fig. 2. The largest contribution to the $\mathrm{S} / \mathrm{N}$ comes from $l \leq 50$, after which the significance reaches a plateau. The $\mathrm{S} / \mathrm{N}$ is highest for $857 \mathrm{GHz}$ and reaches a value of 6.73. Although the CIB $\times$ ISW cross-correlation signal is stronger for the $3000 \mathrm{GHz}$ frequency than at 217,353 , and $545 \mathrm{GHz}$ at $\ell<100$, the $\mathrm{S} / \mathrm{N}$ is lower than that at these frequencies. This can be explained 
Table 2. Predicted S/N for the ISW signal in the ideal scenario (CIB maps free of galactic dust residuals), for $100 \%$ and $40 \%$ sky coverage.

\begin{tabular}{cccccc}
\hline \hline Freq. $(\mathrm{GHz})$ & 217 & 353 & 545 & 857 & 3000 \\
\hline $100 \% f_{\text {sky }}$ & 5.25 & 5.73 & 6.12 & 6.73 & 5.01 \\
$40 \% f_{\text {sky }}$ & 3.32 & 3.62 & 3.87 & 4.26 & 3.17 \\
\hline
\end{tabular}

Notes. The best-fit frequency for the ISW detection in this case is $857 \mathrm{GHz}$, where the $\mathrm{S} / \mathrm{N}$ reaches 6.73 for the full sky and 4.26 for $40 \%$ of the sky. Equation (19) shows that the $\mathrm{S} / \mathrm{N}$ is directly proportional to $\left(f_{\text {sky }}\right)^{0.5}$ and thus can be easily calculated for other sky fractions in this ideal case.

by considering the value of the noise given by Eq. (18). The noise term for the $3000 \mathrm{GHz}$ frequency is much higher at larger scales than it is for other frequencies and hence decreases the $\mathrm{S} / \mathrm{N}$. Therefore, in this ideal scenario, the optimal frequency for the ISW detection is $857 \mathrm{GHz}$. However, in reality, we have to account for sources of noise and contamination in our analysis as well as for reduced sky fractions.

Realistic case. As described earlier, in reality, we have to compromise on certain assumptions made in the previous case. In the ideal case, we assumed that the $\mathrm{CIB} \times \mathrm{ISW}$ cross-correlation signal can be extracted over the whole sky. In a large part of the sky, however, we have strong emissions from our own galaxy. This emission, which is due to the galactic dust, is much higher than the CIB signal. This prevents us from extracting any CIB signal in regions of the sky that are too close to the galactic plane. This directly reduces the available sky fraction for measurement by $\sim 30 \%$. Even then, in the remaining part of the sky, the power spectrum measurements are contaminated by dust that needs to be removed from the maps. This removal will leave some residual noise that has to be accounted for in the analysis. In addition, at lower frequencies, the CMB starts to dominate the CIB. Therefore, the CMB contribution has also to be removed from the maps. At the scales we are interested in, the CMB is limited by cosmic variance and the instrument noise in the CMB measurements can be ignored. When we account for all these effects, the $\mathrm{S} / \mathrm{N}$ becomes

$\left[\frac{S}{N}\right]^{2}(v)=\sum_{\ell=2}^{\ell_{\max }}(2 \ell+1) \frac{f_{\mathrm{sky}}\left[C_{\ell}^{\mathrm{cr}}(v)\right]^{2}}{\left[C_{\ell}^{\mathrm{cr}}(v)\right]^{2}+\left[C_{\ell}^{\mathrm{CIB}}(v)+N_{\ell}^{\mathrm{CIB}}(v)\right] C_{\ell}^{\mathrm{CMB}}}$,

where the $N_{\ell}^{\mathrm{CIB}}(v)$ term contains the dust residuals left in the CIB maps and $f_{\text {sky }}$ represents the fraction of the sky where CMB and CIB are both available. The presence of dust residuals does not affect the measurement of the $\mathrm{CIB} \times \mathrm{CMB}$ cross-correlation as they are not correlated with the CMB.

To quantify the dust residuals in the $\mathrm{CIB}$, that is, $N_{\ell}^{\mathrm{CIB}}(v)$, we used the following procedure:

We computed the dust power spectra using the dust map provided by the PLA ${ }^{1}$ (Planck Legacy Archive) generated using the COMMANDER component separation code (Planck Collaboration X 2016). This map is provided at the reference frequency of $545 \mathrm{GHz}$. In order to account for the available sky fractions, we applied different masks to this map, also provided by PLA. We performed our analysis on $20 \%, 40 \%, 60 \%$, $70 \%$, and $80 \%$ of the sky. We also considered the $10 \%$ mask

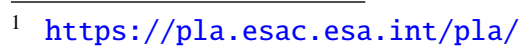

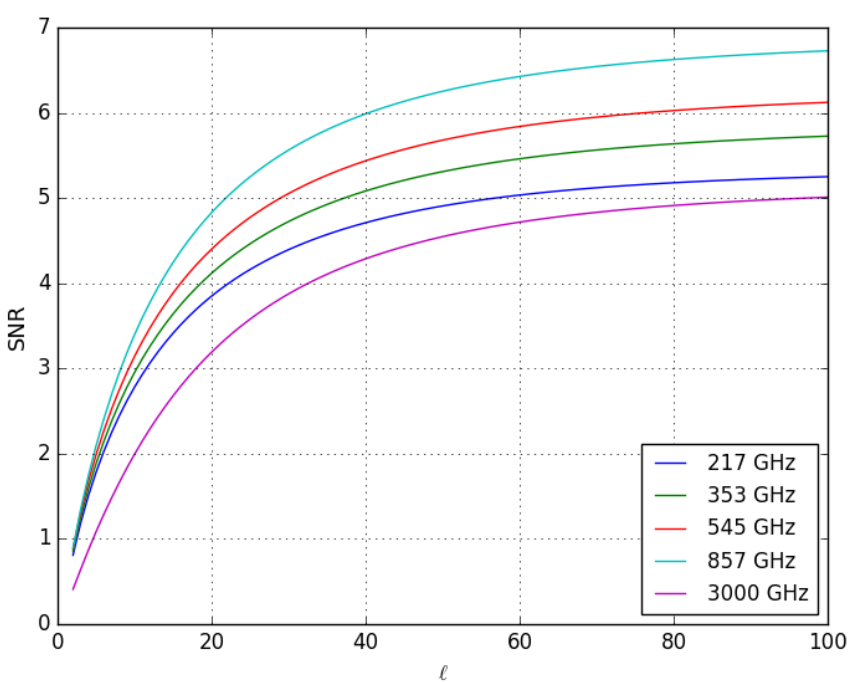

Fig. 2. Cumulative $S / N$ for the $C I B \times I S W$ cross-correlation signal for the five frequency channels. The $\mathrm{S} / \mathrm{N}$ is predicted to be highest for $857 \mathrm{GHz}$

provided in Table 2 of Planck Collaboration XXX (2014). After applying these masks, we computed the dust power spectrum from the dust map using the Xpol software, which is an extension to the polarisation of the Xspect method (Tristram et al. 2005). The maps were analysed with the HEALPix package (Górski et al. 2005).

The power spectrum of the dust $C_{\ell}^{\text {dust }}$ is frequency dependent. After we obtained the power spectrum of the dust at $545 \mathrm{GHz}$, we converted it into other frequencies assuming a modified blackbody shape for dust emission with a given average dust temperature $\left\langle T_{\mathrm{d}}\right\rangle$ and spectral index $\left\langle\beta_{\mathrm{d}}\right\rangle$. The values for $\left\langle T_{\mathrm{d}}\right\rangle$ and $\left\langle\beta_{\mathrm{d}}\right\rangle$ were taken from Table 3 of Planck Collaboration XI (2014) for $|b|>15^{\circ}$ and are $\left\langle T_{\mathrm{d}}\right\rangle=20.3 \mathrm{~K}$ and $\left\langle\beta_{\mathrm{d}}\right\rangle=1.59$.

We briefly explain in Sect. 5.1 that the HI gas column density is observed to be proportional to the galactic dust emission. This relation was used to clean the CIB maps from dust contamination. Dust residuals left in the CIB maps using the HI gas as a dust tracer have been quantified by Planck Collaboration XXX (2014), as detailed in their Sects. 3.2.2 and 3.2.3. They simulated multiple maps of the dust emissivity and obtained the corresponding dust map for a given frequency by multiplying the dust emissivity maps by the GASS map of HI column densities. These dust maps were then used to create simulated maps of total sky emission at all frequencies (e.g., including CIB). Using these sky maps and performing the cleaning of the dust through the above mentioned galactic dust-HI gas column density relation, Planck Collaboration XXX (2014) found the dust residuals on the power spectrum to be of the order of 5-10\% for all the frequencies $(5 \%$ for $217 \mathrm{GHz}$ and $10 \%$ at $857 \mathrm{GHz}$ ). Thus, and to be conservative, we considered here a residual dust power spectrum of $10 \%$ of $C_{\ell}^{\text {dust }}(v)$ at all the frequencies. Therefore, $N_{\ell}^{\mathrm{CIB}}(v)=0.1 \times C_{\ell}^{\mathrm{dust}}(v)$.

After calculating the dust power spectra and eventually $N_{\ell}^{\mathrm{CIB}}(v)$, we calculated the new S/N using Eq. (19). The results are presented in Table 3 . The $\mathrm{S} / \mathrm{N}$ decreases quite dramatically when the dust residuals are included in the analysis. Moreover, the $\mathrm{S} / \mathrm{N}$ decreases when the available sky fraction for the analysis is inreased, which is the exact opposite to the ideal dust-free scenario, where the higher sky fraction resulted in a higher $\mathrm{S} / \mathrm{N}$. The impact of the dust on the CIB power spectra is shown in Fig. 3, where we plot the CIB and the $10 \%$ residual dust power 
Table 3. Predicted S/N for the ISW signal in the current realistic scenario, including the dust residuals in the CIB maps.

\begin{tabular}{cccccc}
\hline \hline Freq. $(\mathrm{GHz})$ & 217 & 353 & 545 & 857 & 3000 \\
\hline $10 \% f_{\text {sky }}$ & 0.88 & 0.91 & 0.83 & 0.65 & 0.28 \\
$20 \% f_{\text {sky }}$ & 1.46 & 1.51 & 1.41 & 1.14 & 0.52 \\
$40 \% f_{\text {sky }}$ & 1.16 & 1.16 & 0.99 & 0.69 & 0.27 \\
$60 \% f_{\text {sky }}$ & 0.92 & 0.90 & 0.74 & 0.49 & 0.18 \\
$70 \% f_{\text {sky }}$ & 0.80 & 0.78 & 0.63 & 0.42 & 0.16 \\
$80 \% f_{\text {sky }}$ & 0.59 & 0.57 & 0.45 & 0.30 & 0.11 \\
\hline
\end{tabular}

Notes. They are given for 10, 20, 40, 60, 70, and $80 \%$ of the sky. These $\mathrm{S} / \mathrm{Ns}$ have been calculated assuming that there is $10 \%$ residual dust in the CIB maps. No signal with a good S/N is detected for the ISW effect in these cases. The best-case scenario seems to be for the $353 \mathrm{GHz}$ frequency over $20 \%$ sky, where the $\mathrm{S} / \mathrm{N}$ reaches 1.51 .

spectra at 353 and $3000 \mathrm{GHz}$. The residual dust power spectra were calculated over $20,40,60,70$, and $80 \%$ of the sky. At the lower multipoles, that is, $\ell<100$ where we expect the ISW signal to be detected, the CIB spectra are completely dominated by the dust residuals. This significantly affects the $\mathrm{S} / \mathrm{N}$ of the ISW signal and decreases it. The highest $\mathrm{S} / \mathrm{N}$ is obtained for $353 \mathrm{GHz}$ with $20 \%$ of the sky fraction available for the analysis. Equation (19) shows that a smaller sky fraction results in decreasing the $\mathrm{S} / \mathrm{N}$ for the ISW detection in the case of dust-free spectra. At the same time, it is clear from Fig. 3 that the residual dust power decreases with smaller sky fractions and hence increases the $\mathrm{S} / \mathrm{N}$. This shows that there is a trade-off between the available sky fraction $f_{\text {sky }}$ and the $\mathrm{S} / \mathrm{N}$ for all the frequencies, and in this case, a $20 \%$ sky fraction provides the best $\mathrm{S} / \mathrm{N}$ for $353 \mathrm{GHz}$.

\section{Forecasting constraints on cosmological parameters}

We showed in Sect. 3.2 that in the ideal scenario of the dust free CIB and CMB maps, the CIB $\times$ ISW cross-correlation can provide us with the detection of the ISW effect with the highest $\mathrm{S} / \mathrm{N}$ for a single tracer. Unfortunately, we are currently limited by the dust residuals in the CIB, which reduces our ability of measuring the ISW signal with a good $\mathrm{S} / \mathrm{N}$. However, in the near future, improved cleaning techniques, additional datasets, and a better understanding of contaminants could allow us to improve the CIB maps, with much lower levels of dust residuals. In such conditions, the $\mathrm{CIB} \times \mathrm{ISW}$ cross-correlation would again become a very competitive probe of cosmology and dark energy in particular. Exploiting the $\mathrm{CIB} \times \mathrm{CMB}$ lensing cross-correlation as well could push the constraints on cosmological parameters even further. With the prospect of these future improved datasets in mind, we perform in this section a joint Fisher matrix analysis including the $\mathrm{CMB}$, the $\mathrm{CIB}$, the $\mathrm{CIB} \times \mathrm{CMB}$ lensing cross-correlation, and the ISW effect through the $\mathrm{CIB} \times \mathrm{CMB}$ cross-correlation and ignoring dust residuals in the CIB.

\subsection{Fisher matrix formalism}

We here recall briefly the principles underlying the Fisher matrix formalism. We consider a set of hypothetical $N$ Gaussian data $\mathbf{x}$ with mean $\boldsymbol{\mu}$ and covariance matrix $\boldsymbol{\Sigma}$ distributed according to the likelihood

$$
L=\frac{1}{(2 \pi)^{n / 2} \sqrt{\operatorname{det} \boldsymbol{\Sigma}}} \exp \left[-\frac{1}{2}(\mathbf{x}-\boldsymbol{\mu})^{T} \boldsymbol{\Sigma}^{-1}(\mathbf{x}-\boldsymbol{\mu})\right] .
$$

Here both $\boldsymbol{\mu}$ and $\boldsymbol{\Sigma}$ depend on $\boldsymbol{\theta}=\left[\theta_{1}, \theta_{2}, \ldots\right]$, the vector of parameters of the model we try to constrain. We are able to forecast the constraints on these parameters (which the hypothetical dataset would provide) in the form of a covariance matrix $\mathbf{C}$, obtained by inverting the so-called Fisher matrix F. The elements of this matrix are defined as the following expectation value:

$F_{m, n}=\left\langle\frac{\partial^{2}(-\ln L)}{\partial \theta_{m} \partial \theta_{n}}\right\rangle$

which after some work, can be rewritten as

$F_{m, n}=\frac{\partial \boldsymbol{\mu}}{\partial \theta_{m}} \boldsymbol{\Sigma}^{-1} \frac{\partial \boldsymbol{\mu}^{T}}{\partial \theta_{n}}+\frac{1}{2} \operatorname{tr}\left(\boldsymbol{\Sigma}^{-1} \frac{\partial \boldsymbol{\Sigma}}{\partial \theta_{m}} \boldsymbol{\Sigma}^{-1} \frac{\partial \boldsymbol{\Sigma}}{\partial \theta_{m}}\right)$,

where $\operatorname{tr}()$ denotes the trace of a square matrix.

In our work, we fixed the covariance matrix to its fiducial value (a common assumption in Fisher analyses) and hence the second term in the Eq. (22) vanishes as $\frac{\partial \boldsymbol{\Sigma}}{\partial \theta_{m}}=0$ and therefore

$F_{m, n}=\frac{\partial \boldsymbol{\mu}}{\partial \theta_{m}} \boldsymbol{\Sigma}^{-1} \frac{\partial \boldsymbol{\mu}^{T}}{\partial \theta_{n}}$

When needed, we computed the partial derivatives as

$\frac{\partial \boldsymbol{\mu}}{\partial \theta_{m}}=\frac{\boldsymbol{\mu}\left(\boldsymbol{\theta}^{\prime}, \theta_{m}+\delta \theta_{m}\right)-\boldsymbol{\mu}\left(\boldsymbol{\theta}^{\prime}, \theta_{m}-\delta \theta_{m}\right)}{2 \delta \theta_{m}}$,

where for a given parameter $\theta_{m}$, the vector $\boldsymbol{\theta}^{\prime}$ represents all the other parameters that are kept constant at their fiducial value. The value of $\delta \theta$ is chosen small enough to stay in the Gaussian regime of the likelihood, but also large enough to not be affected by the numerical errors.

More practically, in our analysis, the parameters of the model are

$\boldsymbol{\theta}=\left[\boldsymbol{\theta}_{\mathrm{CIB}}, \boldsymbol{\theta}_{\mathrm{cosmo}}\right]$

where

$\boldsymbol{\theta}_{\mathrm{CIB}}=\left[\alpha, \beta, \gamma, \delta, b_{0}, b_{1}, b_{2}, f_{v_{i}}^{\mathrm{cal}}\right]$

are the seven CIB model parameters, plus five calibration factors $f_{v_{i}}^{\mathrm{cal}}$ (one for each frequency), which account for relative absolute calibration uncertainties between the different frequency channels. On the other hand,

$\boldsymbol{\theta}_{\text {cosmo. }}=\left[H_{0}, \Omega_{b} h^{2}, \Omega_{c} h^{2}, \tau, n_{s}, A_{s}\right]$

are the $\Lambda$ CDM model parameters, to which we add $w$ when we consider the $w \mathrm{CDM}$ model. Then, in our case, the vector $\boldsymbol{\mu}(\boldsymbol{\theta})$ contains the predicted values of our $N$ observables of interest as a function of our model parameters, and $\boldsymbol{\Sigma}(\boldsymbol{\theta})$ is their theoretical covariance matrix.

As described before, we considered the four following probes as observables: the $\mathrm{CMB}$ power spectra, the $\mathrm{CIB}$ power spectra (+ external constraints), the $\mathrm{CIB} \times \mathrm{CMB}$ lensing crossspectra, and the $C I B \times I S W$ cross-spectra. Our choices for the properties of these observables allowed us to simplify the Fisher analysis. We used the CMB as a prior in our work, and thus neglected its correlation with the other three probes. We also defined our $\mathrm{CIB}$ and $\mathrm{CIB} \times \mathrm{CMB}$ lensing power spectra similarly to the actual data used in Maniyar et al. (2018), meaning that 


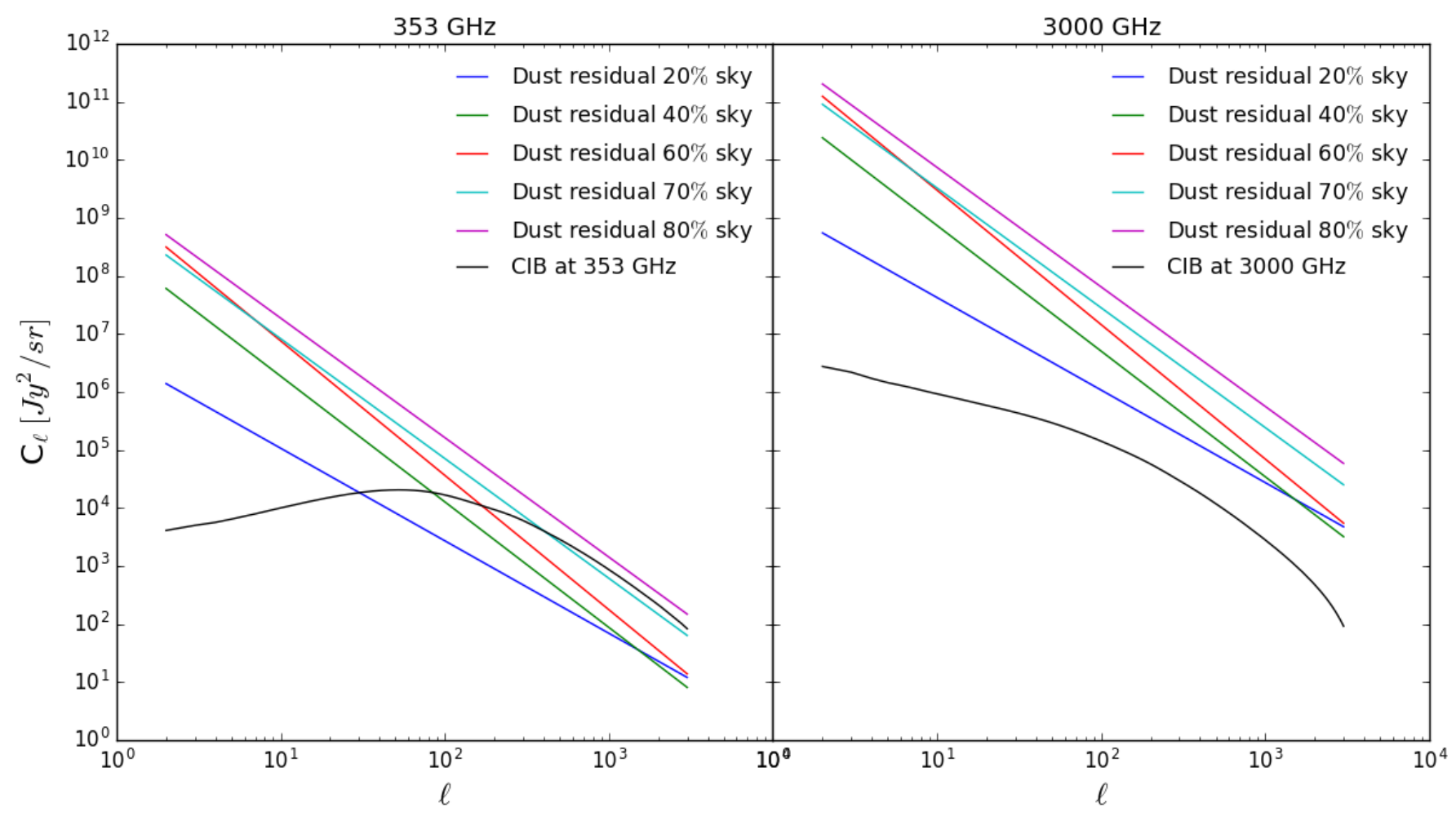

Fig. 3. Power spectra of the CIB and $10 \%$ of the dust residual at $353 \mathrm{GHz}$ (left panel) and $3000 \mathrm{GHz}$ (right panel). The dust power spectra are computed over $20,40,60,70$, and $80 \%$ of the sky. The residual dust power spectra dominate the CIB at the low multipoles of interest for the ISW signal. As a consequence, the $\mathrm{S} / \mathrm{N}$ decreases dramatically compared to the ideal dust-free case.

the spectra were binned in multipoles ${ }^{2}$. This allowed us firstly to ignore the correlations between bins for a given probe, and secondly to neglect correlations between the probes as their respective bins barely overlap. Finally, we can also safely ignore the correlation between the CIB $\times I S W$ spectra and the previous two, as their multipole ranges do not overlap $(\ell \in[2,100])$. Since we did not need to consider the correlations between our different probes, we can write our total Fisher matrix as the sum of the individual Fisher matrices of each probe:

$\mathbf{F}=\mathbf{F}_{\mathrm{CMB}}+\mathbf{F}_{\mathrm{CIB}+\text { ext. }}+\mathbf{F}_{\mathrm{CIB} \times \mathrm{CMB} \text { lensing }}+\mathbf{F}_{\mathrm{CIB} \times \mathrm{ISW}}$.

In the following sections, we explain the construction of the Fisher matrices of the individual probes.

\subsubsection{CMB Fisher matrix}

Our aim is to explore the relative improvement on the constraints on the cosmological parameters set by the CMB using the ISW signal detected through the $\mathrm{CIB} \times \mathrm{CMB}$ cross-correlation. For this reason, we treated the CMB as prior information on all cosmological parameters and used the latest Planck data (plus some external data in the $w C D M$ case, see Sect. 4.3) as constraints. Instead of computing the theoretical $\boldsymbol{\mu}(\boldsymbol{\theta})$ and $\boldsymbol{\Sigma}(\boldsymbol{\theta})$ for the CMB power spectra, we directly derived an equivalent CMB Fisher matrix from Planck data using the official MCMC chains that are publicly available on the $\mathrm{PLA}^{3}$ by computing their covariance

\footnotetext{
2 More precisely, we considered three multipole bins for the CIB power spectra between $\ell \in[145,592]$ and 15 bins for the CIB $\times \mathrm{CMB}$ lensing spectra between $\ell \in[100,2000]$.

3 We used the "plikHM_TTTEEE_lowTEB" chains for the $\Lambda$ CDM model and the "plikHM_TTTEEE_lowTEB_BAO_HO70p6_JLA" chains for the $w$ CDM model.
}

matrix (using the GetDist package provided by Antony Lewis) and inverting it. Since the CMB does not provide any information about the CIB model parameters, the corresponding rows and columns of the CMB Fisher matrix were set to 0 .

\subsubsection{CIB-related Fisher matrix}

In addition to the $\mathrm{CMB}$ prior, we then added as much information as possible to constrain our CIB model parameters. To do so, we considered for our observable vector $\boldsymbol{\mu}$ the same CIB probes as those used as data in Maniyar et al. (2018). These include the CIB auto and cross power spectra, the mean level of the CIB at different frequencies, SFR measurements, and the $\mathrm{CIB} \times \mathrm{CMB}$ lensing cross-correlation. The theoretical value of all these observables for any set of parameters can be computed using the models described earlier in Sects. 2.1 and 2.2. Their derivatives with respect to our model parameters were computed using Eq. (24).

For these observables, instead of computing an analytical covariance matrix $\boldsymbol{\Sigma}$, which may lack subtle features that are present in the real signal (effects of the foreground removals, partial sky coverage, etc.), we used the available CIB data and directly used the estimated covariance matrix of the data from Planck Collaboration XXX (2014) as our fiducial $\Sigma$. As described at the beginning of Sect. 4.1, all CIB observables were considered independent, therefore only the diagonal blocks of the covariance matrix $\boldsymbol{\Sigma}$ are non-zero. This allowed us to compute their respective Fisher matrices independently (using Eq. (22)) and to sum them afterwards. We considered two hypothetical scenarios: one optimistic scenario, where we obtained a CIB map over $f_{\text {sky }}=70 \%$ of the sky, and a more pessimistic one with $f_{\text {sky }}=40 \%$. We multiplied the $\boldsymbol{\Sigma}$ data covariance from Planck Collaboration XXX (2014), 
Table 4. Forecast constraints on $\Lambda$ CDM cosmological parameters.

\begin{tabular}{|c|c|c|c|c|c|c|c|c|c|}
\hline \multicolumn{10}{|c|}{$\Lambda \mathrm{CDM}$ model, $f_{\text {sky }}=70 \%$ case } \\
\hline \multirow{4}{*}{$\mathrm{CMB}+\mathrm{CIB}$} & \multirow{4}{*}{$+\mathrm{CIB} \times \mathrm{CMB}$ lensing } & & $\sigma\left(H_{0}\right)$ & $\sigma\left(\Omega_{b} h^{2}\right)$ & $\sigma\left(\Omega_{c} h^{2}\right)$ & $\sigma(\tau)$ & $\sigma\left(n_{s}\right)$ & $\sigma\left(10^{9} A_{s}\right)$ & $\sigma\left(\Omega_{\Lambda}\right)$ \\
\hline & & & 0.344 & 0.000121 & 0.000812 & 0.0162 & 0.00389 & 0.0710 & 0.00474 \\
\hline & & & $0.574 \%$ & $0.535 \%$ & $0.496 \%$ & $33.9 \%$ & $1.73 \%$ & $36.4 \%$ & $0.538 \%$ \\
\hline & & $+\mathrm{CIB} \times \mathrm{ISW}$ & $19.2 \%$ & $39.0 \%$ & $17.0 \%$ & $38.7 \%$ & $8.05 \%$ & $34.5 \%$ & $17.4 \%$ \\
\hline \multicolumn{10}{|c|}{$\Lambda \mathrm{CDM}$ model, $f_{\text {sky }}=40 \%$ case } \\
\hline \multirow{4}{*}{$\mathrm{CMB}+\mathrm{CIB}$} & & & $\sigma\left(H_{0}\right)$ & $\sigma\left(\Omega_{b} h^{2}\right)$ & $\sigma\left(\Omega_{c} h^{2}\right)$ & $\sigma(\tau)$ & $\sigma\left(n_{s}\right)$ & $\sigma\left(10^{9} A_{s}\right)$ & $\sigma\left(\Omega_{\Lambda}\right)$ \\
\hline & & & 0.412 & 0.000128 & 0.000950 & 0.0164 & 0.00410 & 0.0715 & 0.00565 \\
\hline & $+\mathrm{CIB} \times \mathrm{CMB}$ lensing & & $0.485 \%$ & $0.410 \%$ & $0.458 \%$ & $24.4 \%$ & $1.24 \%$ & $26.3 \%$ & $0.474 \%$ \\
\hline & & $+\mathrm{CIB} \times \mathrm{ISW}$ & $18.1 \%$ & $31.5 \%$ & $15.3 \%$ & $34.2 \%$ & $8.19 \%$ & $31.3 \%$ & $16.4 \%$ \\
\hline
\end{tabular}

Notes. The upper and lower tables show our results in the $f_{\text {sky }}=70 \%$ and $f_{\text {sky }}=40 \%$ cases, respectively. In each table, the first line shows the predicted $1 \sigma$ errors from $\mathrm{CMB}+\mathrm{CIB}$ constraints. The second line shows the improvements on these errors (i.e. relative reduction in percent) after adding the $\mathrm{CIB} \times \mathrm{CMB}$ lensing constraints. The last line shows the further improvement thanks to $\mathrm{CIB} \times \mathrm{ISW}$ constraints, using the $\{\mathrm{CMB}+\mathrm{CIB}+\mathrm{CIB} \times \mathrm{CMB}$ lensing $\}$ case as a reference. The constraints on $\Omega_{\Lambda}$ are derived from the other parameters (and are not originally part of the Fisher analysis).

which was obtained over $10 \%$ of the sky, by a factor of $0.1 / f_{\text {sky }}$ to account for this hypothetical improvement of the CIB recovery.

\subsubsection{CIB $\times I S W$ Fisher matrix}

We considered here as observables the CIB $\times$ ISW crosscorrelation power spectra for all five observed CIB frequencies of Maniyar et al. (2018) and multipoles between $\ell \in[2,100]$. To emulate a future analysis of actual data, and similarly to the CIB power spectra, we chose here to work with binned CIB $\times$ ISW cross-correlation power spectra, splitting the multipole range into 11 roughly equal bins. For a given $\left[\ell_{1}, \ell_{2}\right]$ bin, we defined the binned spectrum $B_{\ell_{1}, \ell_{2}}$ as a simple arithmetic mean,

$B_{\ell_{1}, \ell_{2}}=\frac{1}{n_{\ell}} \sum_{\ell \in\left[\ell_{1}, \ell_{2}\right]} C_{\ell}$

where $n_{\ell}$ is the number of multipoles in the bin, and $C_{\ell}$ in this case is the CIB $\times$ ISW power spectrum as computed using the model described in Sect. 2.3 and their derivative using Eq. (24).

We computed the covariance of the $\mathrm{CIB} \times \mathrm{ISW}$ power spectrum using a generalization of Eq. (18); the covariance between the cross-spectrum $C_{\ell}^{\mathrm{cr}}\left(v_{1}\right)$ at frequency $v_{1}$ and $C_{\ell}^{\mathrm{cr}}\left(v_{2}\right)$ at $v_{2}$ is written as

$\operatorname{Covar}\left(C_{\ell}^{\mathrm{cr}}\left(v_{1}\right), C_{\ell}^{\mathrm{cr}}\left(v_{2}\right)\right)=\frac{C_{\ell}^{\mathrm{cr}}\left(v_{1}\right) C_{\ell}^{\mathrm{cr}}\left(v_{2}\right)+C_{\ell}^{\mathrm{CIB}}\left(v_{1}, v_{2}\right) C_{\ell}^{\mathrm{CMB}}}{f_{\mathrm{sky}}(2 \ell+1)}$.

Similarly to the CIB observables, we considered an optimistic scenario with a dust-free CIB map available over $f_{\text {sky }}=70 \%$ of the sky, and a more pessimistic one with $f_{\text {sky }}=40 \%$. Combining Eq. (30) with the definition of Eq. (29), we can derive the covariance between the binned cross-correlation $B_{\ell_{1}, \ell_{2}}^{\mathrm{cr}}$ at two frequencies:

$\operatorname{Covar}\left(B_{\ell_{1}, \ell_{2}}^{\mathrm{cr}}\left(v_{1}\right), B_{\ell_{1}, \ell_{2}}^{\mathrm{cr}}\left(v_{2}\right)\right)=\frac{1}{n_{\ell}^{2}} \sum_{\ell \in\left[\ell_{1}, \ell_{2}\right]} \operatorname{Covar}\left(C_{\ell}^{\mathrm{cr}}\left(v_{1}\right), C_{\ell}^{\mathrm{cr}}\left(v_{2}\right)\right)$,

which allows us to obtain the covariance matrix $\Sigma$ that is required to compute the $\mathrm{CIB} \times \mathrm{ISW}$ Fisher matrix, again using Eq. (22).

\subsection{Forecasts for the $\Lambda C D M$ model}

We first present our Fisher forecasts in the context of the $\Lambda \mathrm{CDM}$ model. Table 4 shows the $1 \sigma$ constraints on cosmological parameters in the various scenarios we explored, separating our results for our two choices of $f_{\text {sky }}$. We show these constraints in the $\{\mathrm{CMB}+\mathrm{CIB}\}$ case, and their relative improvements achieved by adding the $\mathrm{CIB} \times \mathrm{CMB}$ lensing alone, and the further improvements after adding the $\mathrm{CIB} \times \mathrm{ISW}$ constraints. Figure 4 shows some of these constraints. These contours have been generated using the CosmicFish software (Raveri et al. 2016). The blue ellipses represent the forecast $1 \sigma$ and $2 \sigma$ confidence contours obtained from the combination of $\mathrm{CMB}, \mathrm{CIB}$, and $\mathrm{CIB} \times \mathrm{CMB}$ lensing information. The red ellipses show the corresponding confidence regions after adding the constraints from the CIB $\times$ ISW signal.

As described before, the ISW effect is a probe of the growth of structures through time (see Eq. (8)), which itself is strongly dependent on the characteristics of the dark energy model. The cosmological constant $\Lambda$ affects cosmology only at fairly late times; coupled with the fact that its energy density has remained low and constant in time, the ISW effect will not show its full potential as a probe in the flat $\Lambda \mathrm{CDM}$ model. The two main parameters governing the growth of structures in the flat $\Lambda \mathrm{CDM}$ model are the dark energy density $\Omega_{\Lambda}$ (or equivalently, the matter density $\Omega_{\mathrm{m}}=1-\Omega_{\Lambda}$ ), and the amplitude of matter fluctuations through the power spectrum amplitude $A_{s}$. We thus expect the constraints on these two parameters to be most improved after the ISW signal is added to the Fisher analysis (see also Coble et al. 1997). This expectation is indeed verified: using the $\{\mathrm{CMB}+\mathrm{CIB}+\mathrm{CIB} \times \mathrm{CMB}$ lensing $\}$ case as a reference, we obtain an improvement of $\sim 17 \%$ on the error bars on $\Omega_{\Lambda}$ for $f_{\text {sky }}=70 \%\left(\sim 16 \%\right.$ for $\left.f_{\text {sky }}=40 \%\right)$ and of $\sim 35 \%(\sim 31 \%)$ on $A_{s}$ when the CIB $\times$ ISW information is added to the Fisher matrix (illustrated in Fig. 4). The degeneracy between $\tau$ and $A_{s}$ is well known (e.g. Planck Collaboration XIII 2016), and it benefits from tightening the $A_{s}$ constraints: we thus obtain a similar improvement of $\sim 39 \%(\sim 34 \%)$ on the $\tau$ parameter after adding the ISW information. Interestingly, Table 4 shows that the relative improvements in all constraints when adding $\mathrm{CIB} \times \mathrm{CMB}$ lensing and ISW information is roughly the same for both $f_{\text {sky }}$ (although the absolute constraints are of course better in the $70 \%$ case.) 


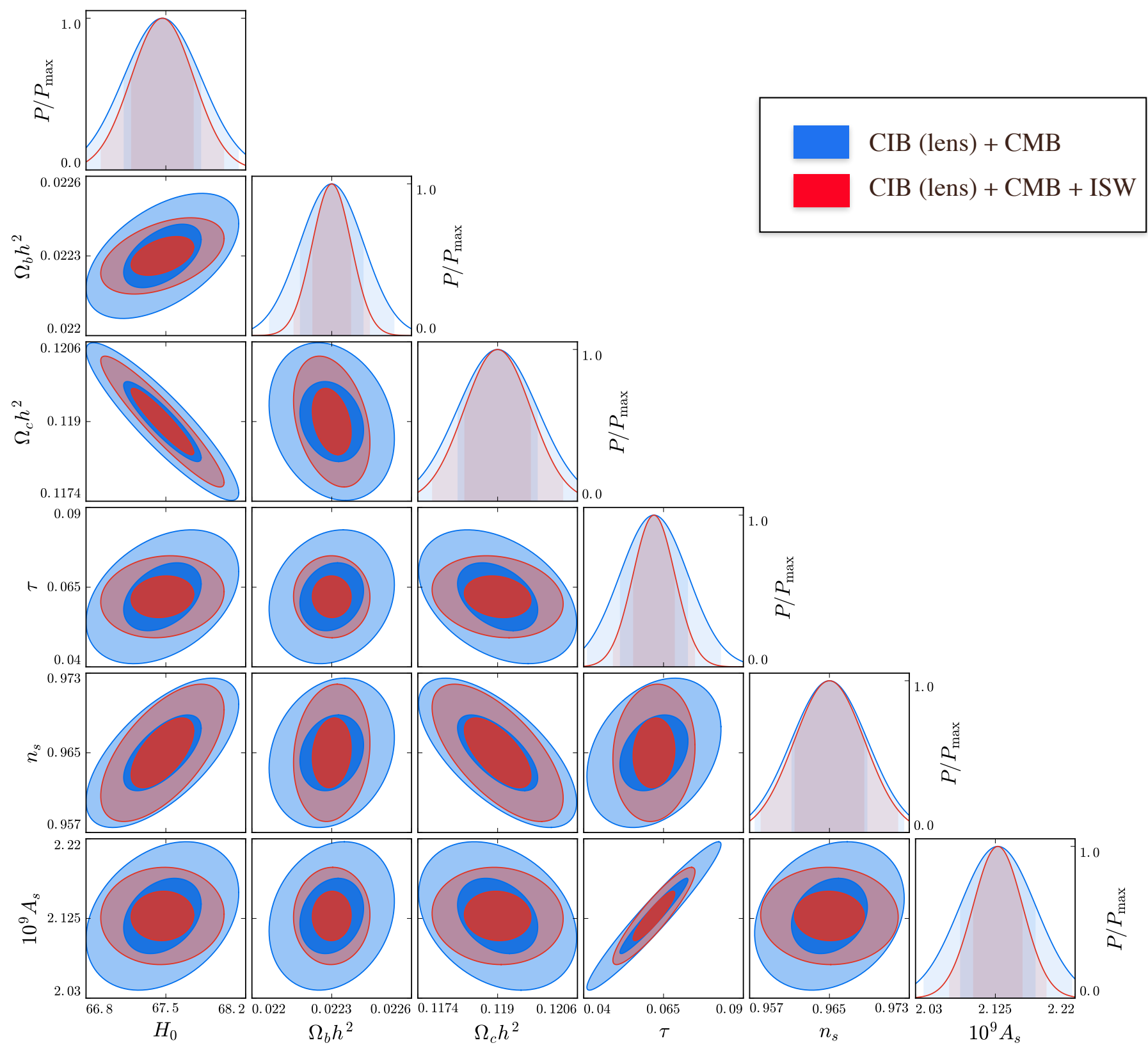

Fig. 4. Fisher forecasts for the $1 \sigma$ and $2 \sigma$ confidence contours for the $\Lambda \mathrm{CDM}$ cosmological parameters in the $f_{\text {sky }}=70 \%$ case. The blue contours show our results when the $\mathrm{CMB}, \mathrm{CIB}$, and $\mathrm{CIB} \times \mathrm{CMB}$ lensing constraints are combined. The red contours show the same results when the CIB $\times$ ISW cross-correlation constraints are added. There is a $\sim 17 \%$ improvement on the constraints on $\Omega_{\Lambda}$ and $\sim 39 / 35 \%$ for $\tau / A_{s}$, which are strongly correlated.

We note that the addition of the CIB $\times$ CMB lensing crosscorrelation alone also improves the constraints on some cosmological parameters. As the CMB lensing signal probes the distribution of matter while being independent of $\tau$ (Planck Collaboration I 2014), we expect it to help break the degeneracy between $\tau$ and $A_{s}$. This is indeed the case, as Table 4 shows that adding the $\mathrm{CIB} \times \mathrm{CMB}$ lensing cross-correlation mainly improves the constraints on these two parameters.

\subsection{Forecasts for the wCDM model}

As pointed out by various studies (e.g. Coble et al. 1997; Corasaniti et al. 2003), the ISW effect has a strong dependence on the equation of state $w \equiv P / \rho$ of the dark energy. This is expected, as any deviation from a pure cosmological constant (i.e. $w \neq-1$ ) has a strong influence on the growth rate of structures at all times, which in turn affect the time variation of the potential wells that the ISW effect probes directly. Combined with the fact that the CIB signal at different frequencies probes the galaxy distribution (and thus the growth of structure) at different redshifts, we thus expect that the addition of the CIB-ISW signal in our Fisher analysis will improve the constraints on $w C D M$ parameters much more than it did for $\Lambda \mathrm{CDM}$. We performed a Fisher analysis for the $w \mathrm{CDM}$ model similar to the case of the $\Lambda \mathrm{CDM}$ model. Here, along with the six cosmological parameters of the $\Lambda \mathrm{CDM}$ model, we have the additional parameter $w$.

The geometrical degeneracy between $w$ and the Hubble constant $H_{0}$ in the context of CMB studies is well known (e.g. Planck Collaboration XIII 2016). This degeneracy arises in order to preserve the angular size of the sound horizon at 

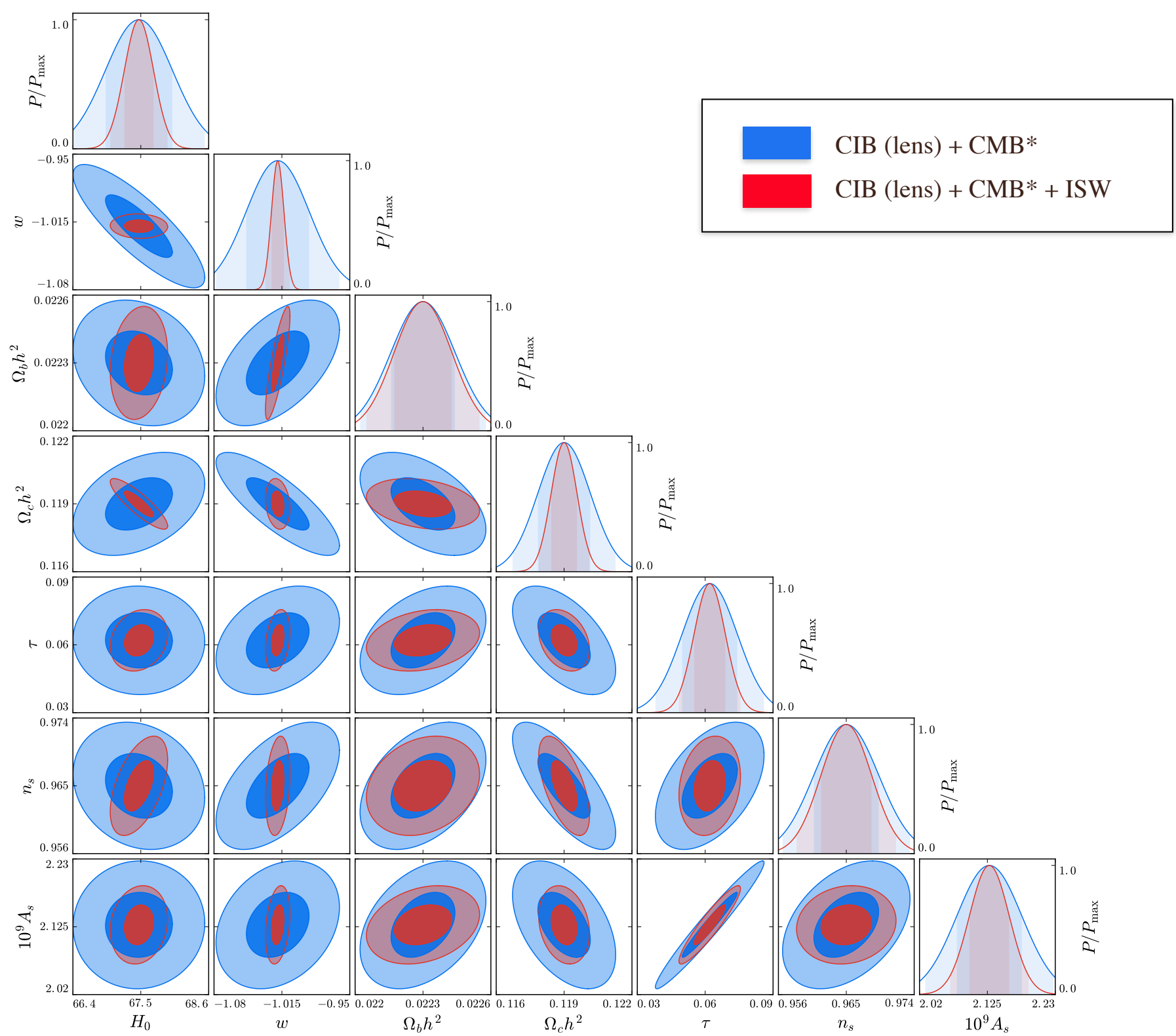

Fig. 5. Fisher forecasts for the $1 \sigma$ and $2 \sigma$ confidence contours for the $w \mathrm{CDM}$ cosmological parameters in the $f_{\text {sky }}=70 \%$ case. The blue contours show our results when the $\mathrm{CIB}, \mathrm{CIB} \times \mathrm{CMB}$ lensing, and the $\mathrm{CMB}$ along with the external (JLA $+\mathrm{BAO}$ and $H_{0}$ prior) constraints on the cosmological parameters are combined. The red contours show the same results when the CIB $\times$ ISW cross-correlation constraints are added. There is a $\sim 80 \%$ improvement on the constraints on $w, \sim 29 \%$ for $\Omega_{\Lambda}$, and $\sim 43 / 39 \%$ for $\tau / A_{s}$, which are strongly correlated.

the last scattering surface. Therefore, the CMB data alone cannot constrain both $H_{0}$ and $w$ together. External cosmological datasets constraining the background evolution of the Universe, such as baryonic acoustic oscillations (BAO), supernovae type Ia (SNe Ia), and local $H_{0}$ measurements, are required to break this degeneracy. In order to extract a valid covariance matrix (and thus deduce an equivalent Fisher matrix) directly from the Planck Markov chain Monte Carlo (MCMC) chains, the corresponding posterior constraints on the cosmological parameters need to follow a Gaussian distribution. This is not the case for the CMB-only chains because of the aforementioned $w-H_{0}$ degeneracy. Therefore, we used the Planck chains provided on the PLA where the CMB data are combined with several BAO datasets (6dFGS, Beutler et al. 2011, SDSS MGS, Ross et al. 2015, BOSS LOWZ and CMASS, Anderson et al. 2014), the JLA supernovae dataset (Betoule et al. 2014), and the local $H_{0}$ measurement from Efstathiou (2014). We refer to the sum of these constraints as $\mathrm{CMB}^{*}$ in the following.

Results for the analysis of the $w \mathrm{CDM}$ model are presented in Fig. 5 and Table 5. Similarly to the $\Lambda$ CDM analysis, the blue and red contours of Fig. 5 represent our forecasts on cosmological parameters without and with CIB-ISW constraints in the Fisher matrix, respectively. We observe a strong improvement on the equation of state $w$ of about $80 \%$ (regardless of $f_{\text {sky }}$ ) when the ISW signal information is added to the Fisher matrix in addition to the $\mathrm{CMB}, \mathrm{CIB}$, and $\mathrm{CIB} \times \mathrm{CMB}$ lensing information. As $w$ is correlated to other cosmological parameters, improvements are partially propagated. This is especially noticeable for $H_{0}$, where an improvement of $\sim 56 \%$ is seen for both $f_{\text {sky }}$. Similarly to the $\Lambda$ CDM case (cf. Sect. 4.2), the ISW signal also improves the constraints on $\Omega_{\Lambda}(\sim 30 \%), A_{s}(\sim 39 / 33 \%)$, and thus $\tau(\sim 43 / 37 \%)$. 
Table 5. Forecast constraints on $w \mathrm{CDM}$ cosmological parameters.

\begin{tabular}{|c|c|c|c|c|c|c|c|c|c|c|}
\hline \multicolumn{11}{|c|}{$w \mathrm{CDM}$ model, $f_{\text {sky }}=70 \%$ case } \\
\hline \multirow{4}{*}{$\mathrm{CMB}^{*}+\mathrm{CIB}$} & & & $\sigma\left(H_{0}\right)$ & $\sigma(w)$ & $\sigma\left(\Omega_{b} h^{2}\right)$ & $\sigma\left(\Omega_{c} h^{2}\right)$ & $\sigma(\tau)$ & $\sigma\left(n_{s}\right)$ & $\sigma\left(10^{9} A_{s}\right)$ & $\sigma\left(\Omega_{\Lambda}\right)$ \\
\hline & & & 0.561 & 0.0308 & 0.000143 & 0.00118 & 0.0170 & 0.00434 & 0.0730 & 0.00480 \\
\hline & $+\mathrm{CIB} \times \mathrm{CMB}$ lensing & & $2.98 \%$ & $2.20 \%$ & $0.577 \%$ & $1.13 \%$ & $27.7 \%$ & $0.756 \%$ & $30.7 \%$ & $2.93 \%$ \\
\hline & & $+\mathrm{CIB} \times \mathrm{ISW}$ & $56.4 \%$ & $80.1 \%$ & $10.1 \%$ & $50.8 \%$ & $43.4 \%$ & $22.0 \%$ & $38.7 \%$ & $29.4 \%$ \\
\hline \multicolumn{11}{|c|}{$w \mathrm{CDM}$ model, $f_{\mathrm{sky}}=40 \%$ case } \\
\hline \multirow{4}{*}{$\mathrm{CMB}^{*}+\mathrm{CIB}$} & & & $\sigma\left(H_{0}\right)$ & $\sigma(w)$ & $\sigma\left(\Omega_{b} h^{2}\right)$ & $\sigma\left(\Omega_{c} h^{2}\right)$ & $\sigma(\tau)$ & $\sigma\left(n_{s}\right)$ & $\sigma\left(10^{9} A_{s}\right)$ & $\sigma\left(\Omega_{\Lambda}\right)$ \\
\hline & & & 0.656 & 0.0330 & 0.000144 & 0.00121 & 0.0170 & 0.00437 & 0.0730 & 0.00575 \\
\hline & $+\mathrm{CIB} \times \mathrm{CMB}$ lensing & & $2.91 \%$ & $1.85 \%$ & $0.322 \%$ & $0.749 \%$ & $20.2 \%$ & $0.553 \%$ & $22.3 \%$ & $3.15 \%$ \\
\hline & & $+\mathrm{CIB} \times \mathrm{ISW}$ & $55.4 \%$ & $79.5 \%$ & $9.15 \%$ & $43.6 \%$ & $36.5 \%$ & $18.8 \%$ & $33.2 \%$ & $29.8 \%$ \\
\hline
\end{tabular}

Notes. The upper and lower tables show our results in the $f_{\text {sky }}=70 \%$ and $f_{\text {sky }}=40 \%$ cases, respectively. In each table, the first line shows the predicted $1 \sigma$ errors from $\mathrm{CMB}^{*}+\mathrm{CIB}$ constraints. The second line shows the improvements on these errors (i.e. relative reduction in percent) after adding the $\mathrm{CIB} \times \mathrm{CMB}$ lensing constraints. The last line shows the further improvement thanks to CIB $\times$ ISW constraints, using the $\{\mathrm{CMB}+\mathrm{CIB}+\mathrm{CMB} \times \mathrm{CIB}$ lensing $\}$ case as a reference. The constraints on $\Omega_{\Lambda}$ are derived from the other parameters (and are not originally part of the Fisher analysis).

These results illustrate for a simple dark energy model the great potential of the $\mathrm{CMB} \times \mathrm{CIB}$ correlation as a probe to constrain such a model, thanks to the discriminating power of the ISW effect. We can expect it to be of similar potential when further extensions to the standard $\Lambda \mathrm{CDM}$ model are to be constrained.

\section{Measurement of the ISW effect using the Planck maps}

The results shown in the previous sections were obtained assuming more or less idealized scenarios. As seen in Sect. 3.2, including the effects of the dust residuals can significantly lower the $\mathrm{S} / \mathrm{N}$. In practice, the currently available CIB maps contain significant amounts of such residuals, and the error bars on the $\mathrm{CIB} \times \mathrm{CMB}$ signal would therefore be dominated by them, $\mathrm{cf}$. Eq. (19). In this section, we present the constraints we can obtain on the cross-correlation measurement using the current CIB and CMB maps.

\subsection{CIB and CMB Planck maps}

Galactic dust emission is observed to be directly proportional to the HI gas column density in diffuse parts of the sky (e.g. Boulanger et al. 1996). This empirical relation is used to clean the map from dust contamination in these parts of the sky (Planck Collaboration XVIII 2011). We used the CIB maps derived in the GASS field at the Planck HFI frequencies as our CIB input maps (Planck Collaboration XXX 2014). GASS (McClure-Griffiths et al. 2009; Kalberla et al. 2010) is a $21 \mathrm{~cm}$ line survey and is one of the most sensitive, highest angular resolution large-scale galactic $\mathrm{HI}$ emission surveys ever made in the southern portion of the sky. The GASS CIB maps cover almost $11 \%$ of the sky and is one of the cleanest (in terms of dust residuals) large patches of the CIB sky, as discussed in Planck Collaboration XXX (2014) ${ }^{4}$. As described earlier, we

\footnotetext{
4 We preferred using the CIB maps built as detailed in Planck Collaboration XXX (2014) rather than the CIB maps provided by the Planck Collaboration PLA and constructed with the procedure detailed in Planck Collaboration Int. XLIX (2016). This is because the Planck CIB maps, which use the GNILC technique to clean the dust, suffer from an excessive cleaning at the lower multipoles in which we are interested, and this results in CIB power removal at these scales. See the Appendix A for more details.
}

used the HEALPix package to analyse the maps. The angular resolution of the CIB maps is 16.2' (set by the GASS survey angular resolution $)^{5}$.

We used the CMB map provided by PLA generated using the SMICA component separation algorithm (Cardoso et al. 2008). The CMB map is degraded to the same angular resolution of $16.2^{\prime}$ as that of the GASS CIB maps. We applied the GASS field mask (Mask2 in Table 2 of Planck Collaboration XXX 2014) to both the CIB and CMB maps and cross-correlated them using $\mathrm{Xpol}$ at all frequencies. Masking induces correlations between different multipoles, and binning the power spectra helps to reduce these correlations. The measurements were then obtained as binned power spectra with a binning of $\Delta_{\ell}=10$.

Figure E.6 of Planck Collaboration IX (2016) shows that the galactic dust and the CIB residuals in the SMICA CMB maps are quite low $(\leq 0.002 \%)$. Hence, we do not expect a cross-correlation between the SMICA CMB map and the GASS CIB maps due to residual dust or residual CIB in the CMB maps.

Figure 6 shows the observed cross-correlation compared to the theoretical prediction for the ISW signal at 217, 353, 545, and $857 \mathrm{GHz}$. The theoretical prediction for the ISW power spectrum was calculated using Eq. (11). This power spectrum was then used to create a map that was given as an input to Xpol to obtain the binned theoretical prediction for the ISW effect, which is shown as the red curve in Fig. 6. Based on the visual inspection alone, it is hard to say if the observed signal is consistent with the predictions and if so, at what level. In order to understand the observed signal, we performed some tests that are discussed below.

\subsection{Significance with respect to the $\Lambda C D M$ prediction}

In order to calculate the significance of the measured crosscorrelation $\hat{C}_{\ell}^{\mathrm{cr}}$ with respect to the ISW $C_{\ell}^{\mathrm{cr}}$ predicted by the $\Lambda \mathrm{CDM}$ model, we performed the following $\chi^{2}$ test. We estimated the amplitude $A$ such that $A C_{\ell}^{\mathrm{cr}}$ is the best-fit solution to $\hat{C}_{\ell}^{\mathrm{cr}}$, that is, the value that minimizes the following quantity:

$\chi^{2}(A)=\left[\hat{C}_{\ell}^{\mathrm{cr}}(v)-A C_{\ell}^{\mathrm{cr}}(v)\right]^{T} \operatorname{Cov}_{\mathrm{cr}}^{-1}(v)\left[\hat{C}_{\ell}^{\mathrm{cr}}(v) A C_{\ell}^{\mathrm{cr}}(v)\right]$

5 The CIB maps, obtained with the PR1 Planck data release, have been corrected to the absolute calibration of the PR2 data release. 


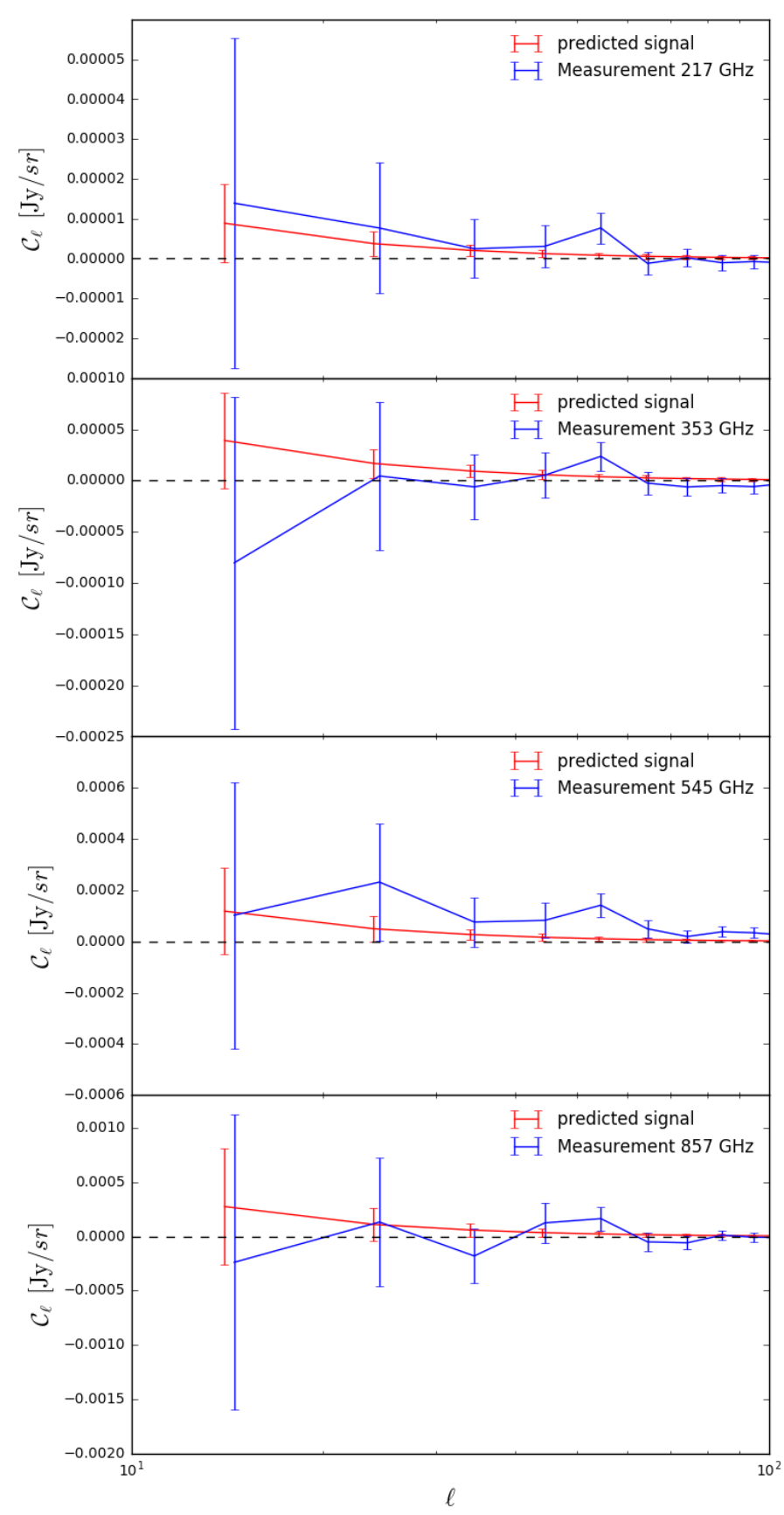

Fig. 6. Measured $\mathrm{CIB} \times \mathrm{CMB}$ cross-correlation and corresponding error bars for the GASS CIB field (blue points). The predicted signal (given by Eq. (11)) is shown in red. The error bars on the predicted signal are calculated using the noise term in Eq. (39). Following Planck Collaboration XXX (2014), we assumed that the CIB power spectrum is contaminated by dust residuals at the level of $10 \%$ when the theoretical error bars were calculated.

where $\operatorname{Cov}_{\mathrm{cr}}(v)$ is the expected covariance matrix on the measured signal. The same minimization also gives us the error $\sigma_{\mathrm{A}}$ on this amplitude. Such a test is convenient, as it informs us about $i$ ) whether the measured signal is compatible with our expectations, that is, $A \sim 1$; and $i$ ) whether the measured signal is significant, that is, $A / \sigma_{\mathrm{A}} \gg 1$.

We used a Monte Carlo approach to compute the error bars for every bin and covariance between different bins (i.e. $\operatorname{Cov}_{\mathrm{cr}}(v)$ ). This approach is similar to that described by Giannantonio et al. (2008) and Serra et al. (2014), for example.
We simulated 1000 pairs of CIB and CMB maps, which were correlated as expected from theory. The expected shot noise and galactic dust residual terms were added to the CIB maps. The detailed procedure is as follows:

- A CIB map for a given frequency is created using the synfast routine from HEALPix. This map is the sum of the pure dust-free CIB term, shot noise, and the dust residual term for the given frequency. Therefore, the total input power spectra to synfast is

$C_{\ell}^{\mathrm{CIB}}(v)=C_{\ell}^{\mathrm{CIB}, \text { pure }}(v)+C_{\ell}^{\mathrm{SN}}+C_{\ell}^{\mathrm{dust}}(v)$,

where $C_{\ell}^{\mathrm{CIB} \text {,pure }}(v), C_{\ell}^{\mathrm{SN}}$ and $C_{\ell}^{\text {dust }}(v)$ are the CIB, shot-noise, and the dust-residual power spectra for a given frequency, respectively. The CIB power spectra are obtained from CAMB. We assumed that after cleaning the GASS field using the dust to gas relation, $10 \%$ of the dust residuals are left. Hence, we took the $10 \%$ of the dust power spectra calculated as described in Sect. 3.2. The shot noise was derived from the Béthermin et al. (2013) model.

- The CMB map is created using the pure CMB term

$C_{\ell}^{\mathrm{CMB}}=C_{\ell}^{\mathrm{CMB}, \text { pure }}$,

where the $C_{\ell}^{\mathrm{CMB} \text {,pure }}$ is obtained from CAMB.

- The CIB and CMB maps are created and correlated using synfast. As explained before, the CIB map is created by adding the maps with the power spectra $C_{\ell}^{\mathrm{CIB}, \text { pure }}(v), C_{\ell}^{\mathrm{SN}}$ and $C_{\ell}^{\text {dust }}(v)$. Using the same seed as was used to create $C_{\ell}^{\text {CIB,pure }}(v)$ map, we created another map with the power spectra $\left(C_{\ell}^{\mathrm{cr}}\right)^{2} / C_{\ell}^{\mathrm{CIB}, \text { pure }}$. Here, $C_{\ell}^{\mathrm{cr}}$ is the predicted crosscorrelation between the CIB and CMB through the ISW effect given by Eq. (11) and is again obtained from CAMB. This second map is added to a third map created using a new seed and with power spectra $C_{\ell}^{\mathrm{CMB}}-\left(C_{\ell}^{\mathrm{cr}}\right)^{2} / C_{\ell}^{\mathrm{ClB}, \text { pure }}$. Finally, these two maps will have amplitudes

$a_{\ell m}^{\mathrm{CIB}}=\xi_{a}\left(C_{\ell}^{\mathrm{CIB}}\right)^{1 / 2}$,

$a_{\ell m}^{\mathrm{CMB}}=\xi_{a}\left(\left(C_{\ell}^{\mathrm{cr}}\right)^{2} / C_{\ell}^{\mathrm{CIB}, \text { pure }}\right)^{1 / 2}+\xi_{b}\left(C_{\ell}^{\mathrm{CMB}}-\left(C_{\ell}^{\mathrm{cr}}\right)^{2} / C_{\ell}^{\mathrm{CIB}, \text { pure }}\right)^{1 / 2}$,

where $\xi$ is a random amplitude with zero mean and unit variance so that $\left\langle\xi \xi^{*}\right\rangle=1$ and $\langle\xi\rangle=0$. This gives us

$$
\begin{aligned}
\left\langle a_{\ell m}^{\mathrm{CIB}} a_{\ell m}^{\mathrm{CIB} *}\right\rangle & =C_{\ell}^{\mathrm{CIB}}, \\
\left\langle a_{\ell m}^{\mathrm{CIB}} a_{\ell m}^{\mathrm{CMB} *}\right\rangle & =C_{\ell}^{\mathrm{cr}}, \\
\left\langle a_{\ell m}^{\mathrm{CMB}} a_{\ell m}^{\mathrm{CMB} *}\right\rangle & =C_{\ell}^{\mathrm{CMB}} .
\end{aligned}
$$

- When these maps were obtained, the GASS field mask covering $\sim 11 \%$ sky was applied to both of them and their crosspower spectra were computed using Xpol. This gives us 1000 sets of cross-spectra for each frequency, which provide us with the uncertainty on our estimate. The covariance matrix for the binned power spectrum is then calculated as

$C_{b b^{\prime}}^{\mathrm{cr}}=\left\langle\left(C_{b}-\left\langle C_{b}\right\rangle_{\mathrm{MC}}\right)\left(C_{b^{\prime}}-\left\langle C_{b^{\prime}}\right\rangle_{\mathrm{MC}}\right)\right\rangle_{\mathrm{MC}}$,

where $\langle$.$\rangle denotes the average of the Monte Carlo samples.$ The error bars on every bin then become

$\sigma_{\mathrm{C}_{b}}=\left(C_{b b}\right)^{1 / 2}$. 
Table 6. Best-fit values and errors for the amplitude $A$, and significance of the signal $A / \sigma_{\mathrm{A}}$ for all the frequencies.

\begin{tabular}{cccccccc}
\hline \hline & \multicolumn{3}{c}{ With $\ell=55$} & & \multicolumn{3}{c}{ Without $\ell=55$} \\
\cline { 2 - 5 } \cline { 6 - 8 } Freq. (GHz) & $A$ & $\sigma_{\mathrm{A}}$ & $A / \sigma_{\mathrm{A}}$ & & $A$ & $\sigma_{\mathrm{A}}$ & $A / \sigma_{\mathrm{A}}$ \\
\hline 217 & 2.03 & 1.12 & 1.8 & & 0.77 & 1.24 & 0.63 \\
353 & -0.19 & 1.12 & -0.17 & -1.22 & 1.22 & -1.0 \\
545 & 6.64 & 1.20 & 5.52 & & 5.53 & 1.34 & 4.12 \\
857 & 0.11 & 1.62 & 0.07 & & -1.17 & 1.80 & -0.65 \\
\hline
\end{tabular}

Notes. They were calculated using $\ell \in[10,100]$, with and without the data point at $\ell=55$ (see text for more details). No significant detection of the signal with respect to the $\Lambda \mathrm{CDM}$ prediction can be claimed based on these values, except for the signal observed at $545 \mathrm{GHz}$.

The error bars calculated in this way can be compared with the analytical estimate:

$\sigma_{\mathrm{C}_{b}}(v)=\left(\frac{C_{b}^{\mathrm{cr}}(v)^{2}+\left(C_{b}^{\mathrm{CIB}}(v)+N_{b}^{\mathrm{CIB}}(v)\right) C_{b}^{\mathrm{CMB}}}{f_{\mathrm{sky}}(2 \ell+1) \Delta \ell}\right)^{1 / 2}$.

We observe that the error bars obtained from the simulations are $22-25 \%$ higher than those obtained with Eq. (39) for different frequencies. This is expected as Eq. (39) is not very accurate for very low sky fractions, which is the case here. We calculated the correlation matrix based on the 1000 simulations for every frequency and found that the correlations between adjacent multipoles are always lower than $16 \%$ (with a binning $\Delta_{\ell}=10$ ).

After we obtained the expected covariance matrix using the above procedure, we used Eq. (32) to minimise the $\chi^{2}$ and fit for the amplitude $A$. The results are shown in Table 6. Except for $545 \mathrm{GHz}$, we see that although the amplitudes of the measured signals are roughly compatible with 1 (within the estimated error bars), the significance of these cross-correlations is quite low. At $545 \mathrm{GHz}, A / \sigma_{\mathrm{A}}$ is equal to 5.52 with $A=6.64$. Therefore, the observed amplitude of the cross-correlation is $(6.64-1) / 1.2=4.7 \sigma$ higher than what is expected from the $\Lambda \mathrm{CDM}$ model. Possible explanations for this high significance are explored in the next subsection.

\subsection{Null test}

The previous test showed that no conclusion can be reached regarding the question whether the measured cross-correlations are consistent with a detection. Therefore, we also checked the significance of the observed signal against the null hypothesis. For this purpose, we performed a null test: for every frequency, we cross-correlated the GASS CIB field map with 1000 randomly generated CMB maps. These 1000 cross-correlations give us the covariance matrix and hence also the error bars for different bins for the null signal. Figure 7 shows the $1 \sigma$ region spanned by this null signal, together with the measured $\mathrm{CIB} \times \mathrm{CMB}$ crosscorrelation at $545 \mathrm{GHz}$.

We performed a $\chi^{2}$ test for the observed cross-correlation signal with the null signal such that

$\chi_{\text {null }}^{2}=\left[\hat{C}_{\ell}^{\mathrm{cr}}(v)\right]^{T} \operatorname{Cov}_{\mathrm{cr}}^{-1}(v)\left[\hat{C}_{\ell}^{\mathrm{cr}}(v)\right]$

where $\hat{C}_{\ell}^{\mathrm{cr}}(v)$ is the observed cross-correlation signal and $\operatorname{Cov}_{\mathrm{cr}}(v)$ is the covariance matrix computed from the simulations as described above for all the frequencies. We obtained a

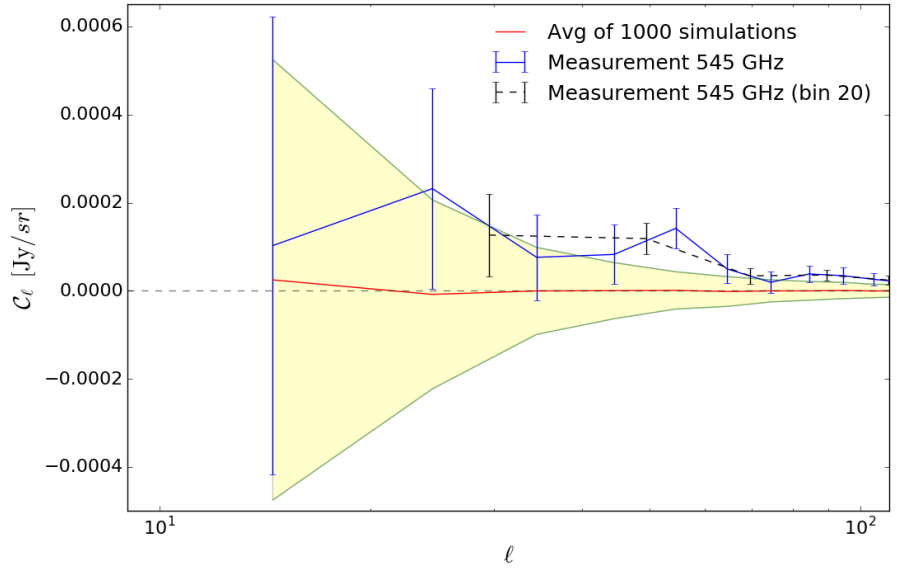

Fig. 7. Average null signal calculated using the cross-correlation of the GASS CIB field with 1000 random CMB maps shown in red. The shaded area in yellow spans the $1 \sigma$ region of the null signal, which is represented by the green lines. The measured $\mathrm{CIB} \times \mathrm{CMB}$ cross-correlation is shown in blue. We also show the $\mathrm{CIB} \times \mathrm{CMB}$ crosscorrelation measured using a bin size $\Delta \ell=20$ instead of 10 (black points and dashed line).

$\chi^{2}$ value of 6.0, 5.6, and 4.9 for 9 degrees of freedom, which corresponds to the $p$-value of $0.74,0.78$ and 0.84 for 217,353 , and $857 \mathrm{GHz}$, respectively, which shows that the signal is compatible with null. For $545 \mathrm{GHz}$, we obtained a $\chi^{2}$ of 30.7 , which corresponds to a $p$-value of 0.0003 . Such a high value of the $\chi^{2}$ is observed because the bump that is observed in the measured cross-correlation near $\ell \approx 55 . \chi^{2}$ decreases to 16.7 with a corresponding $p$-value of 0.03 when this data point is not considered when $\chi^{2}$ is calculated.

We investigated the source of the bump at $\ell \approx 55$. It can have three different origins: residual systematics in the data, correlated residual astrophysical signals (e.g. galactic dust), or a simple $<3 \sigma$ deviation. The bump is observed at all frequencies (from 217 to $857 \mathrm{GHz}$ ), which tends to rule out any systematics left in the data. In the GASS field, it is also present when using the GNILC CIB map (see Appendix A) rather than the Planck 2013 CIB map, and also using different Planck CMB maps (SMICA, but also COMMANDER). In contrast, it is not visible in another field when the cross-correlation is computed using the GNILC CIB map and $11 \%$ of the sky in the northern hemisphere. This tends to show that it cannot be due to a residual astrophysical signal correlated in the CIB and CMB maps, and is thus a feature that is not statistically significant (less than $3 \sigma$ ) in the GASS field.

These tests show that the cross-correlation between the CIB and CMB obtained for the GASS field (with a sky fraction of $11 \%$ ) is compatible with the null signal. CIB maps extracted over larger sky fractions and with lower dust residuals are required to extract the ISW signal using the $\mathrm{CIB} \times \mathrm{CMB}$ cross-correlation.

\section{Conclusions}

We used the CIB as a potential tracer of the large-scale structures to extract the ISW signal in the CMB with a high $\mathrm{S} / \mathrm{N}$. Our approach made use of the framework presented in Ilić et al. (2011). We used the improved linear CIB model of Maniyar et al. (2018), which includes the $\mathrm{CIB} \times \mathrm{CMB}$ lensing cross-correlation while fitting for the data, which partially breaks the degeneracy between the effective bias of the CIB galaxies and their emissivity and provides improved constraints on the CIB model parameters. 
With this improved CIB model, we computed theoretical predictions for the $\mathrm{CIB} \times \mathrm{ISW}$ cross-correlation for different frequencies and found, as expected from the ISW effect, that it peaks at large angular scales, namely $20<\ell<50$ for all the frequencies considered. We showed that this cross-correlation can provide us the ISW signal with the highest $\mathrm{S} / \mathrm{N}$ ever obtained for a single tracer. The optimal frequency with the highest $\mathrm{S} / \mathrm{N}$ for the ISW detection in this case is $857 \mathrm{GHz}$ with an $S / N$ of $6.7 \sigma$. Using a Fisher matrix approach, we calculated the expected improvement on the constraints on the cosmological parameters as set by Planck (+SN, BAO, and $\mathrm{H} 0)$ by adding the ISW signal obtained with the $\mathrm{CIB} \times \mathrm{CMB}$ cross-correlation. This analysis was done for the $\Lambda \mathrm{CDM}$ and the $w C D M$ cosmological models, and we found as expected that the constraints on the latter are more sensitive to the addition of the ISW signal. For that model, we found that the $\mathrm{CIB} \times \mathrm{CMB}$ cross-correlation could potentially improve the constraints on the dark energy density parameter $\Omega_{\Lambda}$ by $\sim 30 \%$ and the equation of state of the dark energy $w$ by $\sim 80 \%$, whether the available fraction of the sky is $70 \%$ or $40 \%$. It also improves the constraints on the $A_{s}$ parameter, as well as $\tau$ due to their degeneracy. The $\mathrm{CIB} \times \mathrm{CMB}$ lensing cross-correlation breaks the degeneracy between $A_{s}$ and $\tau$ and improves the constraints on these parameters. The so-called stage-4 next-generation ground-based CMB experiment (CMB$\mathrm{S} 4$ ) is expected to produce maps of unprecedented quality, improving the $\mathrm{S} / \mathrm{N}$ of the reconstructed lensing map by over an order of magnitude (Abazajian et al. 2016) compared to Planck. While this will naturally improve the $\mathrm{S} / \mathrm{N}$ for the $\mathrm{CIB} \times \mathrm{CMB}$ lensing cross-correlation, we recall that the maximum amount of cosmological information that can be extracted is ultimately limited by cosmic variance. We performed a Fisher matrix analysis to forecast the constraints on the cosmological parameters with the improved $\mathrm{CIB} \times \mathrm{CMB}$ lensing cross-correlation. As expected, compared to current constraints using Planck data, a small improvement (a few percent) is observed for most cosmological parameters and a significant improvement ( 30\%) for $\tau$ and $A_{s}$.

This analysis was performed in relatively idealised scenarios with CIB maps free of any galactic dust residuals, however. To assess the effects of partial sky coverage and galactic dust residuals, we calculated the dust power spectra for all the frequencies over various sky fractions. We find that at our multipoles of interests, that is, $\ell<100$, the residual dust power spectra completely dominate the CIB power spectra. As a result, taking $10 \%$ of the dust power spectra as the possible residuals in our cleaned maps, we performed the same analysis over given portions of the sky and showed that the $\mathrm{S} / \mathrm{N}$ drastically decreases with an $\mathrm{S} / \mathrm{N}$ of 1.5 for $353 \mathrm{GHz}$ over $20 \%$ sky fraction, for instance.

Using CIB and CMB maps obtained by Planck, we attempted to measure the $\mathrm{CIB} \times \mathrm{CMB}$ cross-correlation, allowing us to check the feasibility of detecting the ISW effect with the current level of dust removal and available sky fraction. This crosscorrelation was computed over $\sim 11 \%$ of the southern sky in a field that is one of the largest and cleanest patches of the CIB to date (Planck Collaboration XXX 2014). We calculated the significance of the observed signal with respect to the $\Lambda \mathrm{CDM}$ prediction. We performed Monte Carlo simulations in order to obtain the expected covariance matrix between different bins for a given frequency for this test. We find that for all the frequencies except for $545 \mathrm{GHz}$, the signal is roughly compatible with expectations, but is detected with very low significance. At $545 \mathrm{GHz}$, the signal is detected at $5.5 \sigma$, but at the same time, its amplitude is $4.7 \sigma$ higher than that predicted by the $\Lambda \mathrm{CDM}$ model. We also performed a null test to determine whether the detected signal is compatible with the null hypothesis. Again, except for $545 \mathrm{GHz}$, we find that the signal is consistent with the null hypothesis. For $545 \mathrm{GHz}$, the null hypothesis is rejected with a $p$-value of 0.0003 , with a $\chi^{2}$ of 30.7 for 9 degrees of freedom. When we performed the null test by ignoring a bump observed in the cross-correlation around $\ell \sim 55$, we obtained a $p$-value of 0.03 with a $\chi^{2}$ of 16.7 for 8 degrees of freedom. These tests show that the measured $\mathrm{CIB} \times \mathrm{CMB}$ cross-correlation is compatible with the null hypothesis and that we do not detect any significant signal. Our analysis shows that with the current state of the maps, the ISW signal cannot be detected. A much better cleaning of galactic dust is required. If we do not wish to degrade the $\mathrm{S} / \mathrm{N}$ on the measurements by more than $10 \%$ on $40 \%$ of the sky, we need to clean the dust up to the $0.01 \%$ level on the power spectrum. This is very challenging (if at all possible) to achieve, but mandatory for using the CIB as a cosmological probe either through the ISW effect but also in itself, for example, to constrain local primordial non-Gaussianity or other nonstandard cosmological models. Granett et al. (2008) performed a stacking of CMB patches at the locations of a sample of 100 large voids and galaxy clusters, aiming at measuring their ISW signal. They reported a signal whose amplitude was significantly stronger than expected in the $\Lambda$ CDM model. This work has later on been challenged by other groups, and was extended to larger catalogues of structures (see e.g. Kovács 2018, and references therein for a recent review), with the consensus that the stacking signal appears indeed stronger than expected. If the origin of this discrepancy were to be an excess of ISW signal, it is not straightforward to predict how (or if) it would appear in our present analysis because we work in harmonic space, whereas stacking is done directly in maps. We intend to explore this particular subject in the near future in order to determine if this potential excess ISW signal could be detected using the CIB maps along with the CMB.

Acknowledgements. A.M. and G.L. warmly thank Matthieu Tristram for his invaluable help in the use of Xpol. We acknowledge financial support from the "Programme National de Cosmologie and Galaxies" (PNCG) funded by CNRS/INSU-IN2P3-INP, CEA and CNES, France, from the ANR under the contract ANR-15-CE31-0017 and from the OCEVU Labex (ANR-11-LABX-0060) and the A*MIDEX project (ANR-11-IDEX-0001-02) funded by the "Investissements d'Avenir" French government programme managed by the ANR. The research leading to these results has also received funding from the European Research Council under the European Union's Seventh Framework Programme (FP7/2007-2013)/ERC Grant Agreement No. 617656 "Theories and Models of the Dark Sector: Dark Matter, Dark Energy and Gravity".

\section{References}

Abazajian, K. N., Adshead, P., Ahmed, Z., et al. 2016, ArXiv e-prints [arXiv:1610.02743]

Anderson, L., Aubourg, É., Bailey, S., et al. 2014, MNRAS, 441, 24

Béthermin, M., Wang, L., Doré, O., et al. 2013, A\&A, 557, A66

Béthermin, M., Daddi, E., Magdis, G., et al. 2015, A\&A, 573, A113 Béthermin, M., Wu, H.-Y., Lagache, G., et al. 2017, A\&A, 607, A89 Betoule, M., Kessler, R., Guy, J., et al. 2014, A\&A, 568, A22

Beutler, F., Blake, C., Colless, M., et al. 2011, MNRAS, 416, 3017 Boughn, S. P., \& Crittenden, R. G. 2002, Phys. Rev. Lett., 88, 021302 Boughn, S. P., Crittenden, R. G., \& Turok, N. G. 1998, New Astron., 3, 275 Boulanger, F., Abergel, A., Bernard, J.-P., et al. 1996, A\&A, 312, 256

Cabré, A., Gaztañaga, E., Manera, M., Fosalba, P., \& Castander, F. 2006, MNRAS, 372, L23

Cardoso, J.-F., Le Jeune, M., Delabrouille, J., Betoule, M., \& Patanchon, G. 2008, IEEE J. Sel. Top. Sign. Proces., 2, 735

Coble, K., Dodelson, S., \& Frieman, J. A. 1997, Phys. Rev. D, 55, 1851

Corasaniti, P. S., Bassett, B. A., Ungarelli, C., \& Copeland, E. J. 2003, Phys. Rev. Lett., 90, 091303

Creque-Sarbinowski, C., Bird, S., \& Kamionkowski, M. 2016, Phys. Rev. D, 94 , 063519 
Efstathiou, G. 2014, MNRAS, 440, 1138

Eisenstein, D. J., \& Hu, W. 1998, ApJ, 496, 605

Ferraro, S., Sherwin, B. D., \& Spergel, D. N. 2015, Phys. Rev. D, 91, 083533

Francis, C. L., \& Peacock, J. A. 2010, MNRAS, 406, 2

Garriga, J., Pogosian, L., \& Vachaspati, T. 2004, Phys. Rev. D, 69, 063511

Giannantonio, T., Crittenden, R. G., Nichol, R. C., et al. 2006, Phys. Rev. D, 74, 063520

Giannantonio, T., Scranton, R., Crittenden, R. G., et al. 2008, Phys. Rev. D, 77, 123520

Górski, K. M., Hivon, E., Banday, A. J., et al. 2005, ApJ, 622, 759

Granett, B. R., Neyrinck, M. C., \& Szapudi, I. 2008, ApJ, 683, L99

Hanson, D., Hoover, S., Crites, A., et al. 2013, Phys. Rev. Lett., 111, 141301

Hernández-Monteagudo, C., Ross, A. J., Cuesta, A., et al. 2014, MNRAS, 438, 1724

Ilić, S., Douspis, M., Langer, M., Pénin, A., \& Lagache, G. 2011, MNRAS, 416, 2688

Ilić, S., Langer, M., \& Douspis, M. 2013, A\&A, 556, A51

Kalberla, P. M. W., McClure-Griffiths, N. M., Pisano, D. J., et al. 2010, A\&A, 521, A17

Kennicutt, Jr., R. C. 1998, ARA\&A, 36, 189

Knox, L., Cooray, A., Eisenstein, D., \& Haiman, Z. 2001, ApJ, 550, 7

Kodama, H., \& Sasaki, M. 1984, Prog. Theor. Phys. Suppl., 78, 1

Kovács, A. 2018, MNRAS, 475, 1777

Lagache, G., \& Puget, J. L. 2000, A\&A, 355, 17

Lagache, G., Bavouzet, N., Fernandez-Conde, N., et al. 2007, ApJ, 665, L89

Lewis, A. 2014, http://cosmologist.info/notes/CAMB.pdf

Lewis, A., \& Bridle, S. 2002, Phys. Rev. D, 66, 103511

Lewis, A., \& Challinor, A. 2006, Phys. Rep., 429, 1

Ma, C.-P., \& Bertschinger, E. 1995, ApJ, 455, 7

Madau, P., \& Dickinson, M. 2014, ARA\&A, 52, 415

Maniyar, A. S., Béthermin, M., \& Lagache, G. 2018, A\&A, 614, A39

Matsuhara, H., Kawara, K., Sato, Y., et al. 2000, A\&A, 361, 407

McClure-Griffiths, N. M., Pisano, D. J., Calabretta, M. R., et al. 2009, ApJS, 181,398

Okamoto, T., \& Hu, W. 2003, Phys. Rev. D, 67, 083002
Perlmutter, S., Aldering, G., Goldhaber, G., et al. 1999, ApJ, 517, 565 Pietrobon, D., Balbi, A., \& Marinucci, D. 2006, Phys. Rev. D, 74, 043524 Planck Collaboration XVIII. 2011, A\&A, 536, A18

Planck Collaboration I. 2014, A\&A, 571, A1

Planck Collaboration XI. 2014, A\&A, 571, A11

Planck Collaboration XVIII. 2014, A\&A, 571, A18

Planck Collaboration XXX. 2014, A\&A, 571, A30

Planck Collaboration IX. 2016, A\&A, 594, A9

Planck Collaboration X. 2016, A\&A, 594, A10

Planck Collaboration XIII. 2016, A\&A, 594, A13

Planck Collaboration XXI. 2016, A\&A, 594, A21

Planck Collaboration Int. XLIX. 2016, A\&A, 596, A109

Puget, J.-L., Abergel, A., Bernard, J.-P., et al. 1996, A\&A, 308, L5

Raccanelli, A., Bonaldi, A., Negrello, M., et al. 2008, MNRAS, 386, 2161

Raveri, M., Martinelli, M., Zhao, G., \& Wang, Y. 2016. ArXiv e-prints [arXiv:1606.06268]

Riess, A. G., Filippenko, A. V., Challis, P., et al. 1998, AJ, 116, 1009

Riess, A. G., Macri, L. M., Hoffmann, S. L., et al. 2016, ApJ, 826, 56

Ross, A. J., Samushia, L., Howlett, C., et al. 2015, MNRAS, 449, 835

Sachs, R. K., \& Wolfe, A. M. 1967, ApJ, 147, 73

Scoccimarro, R. 2004, Phys. Rev. D, 70, 083007

Seljak, U. 1996, ApJ, 460, 549

Serra, P., Lagache, G., Doré, O., Pullen, A., \& White, M. 2014, A\&A, 570, A98

Shajib, A. J., \& Wright, E. L. 2016, ApJ, 827, 116

Song, Y.-S., Cooray, A., Knox, L., \& Zaldarriaga, M. 2003, ApJ, 590, 664

Stölzner, B., Cuoco, A., Lesgourgues, J., \& Bilicki, M. 2018, Phys. Rev. D, 97, 063506

Taburet, N., Hernández-Monteagudo, C., Aghanim, N., Douspis, M., \& Sunyaev, R. A. 2011, MNRAS, 418, 2207

Tinker, J. L., Sheldon, E. S., Wechsler, R. H., et al. 2012, ApJ, 745, 16

Tristram, M., Macías-Pérez, J. F., Renault, C., \& Santos, D. 2005, MNRAS, 358, 833

Viero, M. P., Moncelsi, L., Quadri, R. F., et al. 2013a, ApJ, 779, 32

Viero, M. P., Wang, L., Zemcov, M., et al. 2013b, ApJ, 772, 77

Wu, H.-Y., Doré, O., Teyssier, R., \& Serra, P. 2018, MNRAS, 475, 3974 


\section{Appendix A: Choice of the CIB maps}

As described in Sect. 5.1, we used the CIB maps in the GASS field built as detailed in Planck Collaboration XXX (2014), and not the GNILC CIB maps provided by the Planck Collaboration. The Planck CIB maps have been prepared by cleaning the galactic dust using the GNILC component separation method and the details are provided in Planck Collaboration Int. XLIX (2016). Figure 6 of Planck Collaboration Int. XLIX (2016) shows the power spectrum of galactic dust and galactic-dustsubtracted Planck maps (also known as the CIB), together with the best-fit model of the CIB power spectrum from Planck Collaboration XXI (2016) for different frequencies. This figure shows that there is an excessive dust cleaning at 545 and $857 \mathrm{GHz}$ at low multipoles, which are of interest to us, and hence the CIB power spectra from GNILC maps is lying below the Planck Collaboration XXX (2014) best-fit model. Maniyar et al. (2018) provided an improved CIB model, using not only the CIB measurements, but also the $\mathrm{CIB} \times \mathrm{CMB}$ lensing cross-correlation.

To check the validity of the CIB map in the GASS field, we cross-correlated it with the lensing convergence $\kappa$ map (provided by Planck ${ }^{6}$ ) using Xpol. For comparison, we also considered the Planck GNILC CIB map from PLA and crosscorrelated it with the same lensing convergence map. The cross-correlation of $\mathrm{CIB} \times \mathrm{CMB}$ lensing potential $\phi$ is obtained as

$$
C_{\ell}^{\mathrm{CIB} \times \phi}=\frac{C_{\ell}^{\mathrm{CIB} \times \kappa}}{0.5 \ell(\ell+1)} .
$$

Figure A.1 shows the $\ell^{3} C_{\ell}^{\mathrm{CIB} \times \phi}$ computed both for GASS CIB map and Planck GNILC CIB map for $545 \mathrm{GHz}$ till $\ell=100$. The cross-correlation using the GASS CIB map falls on the Maniyar et al. (2018) model, whereas the cross-correlation with the Planck GNILC CIB map is consistently lower (by a factor of $>2$ at $\ell=50$ ) in this range of multipoles in which we are interested in for ISW. At the same time, the error bars on the crosscorrelation with the Planck GNILC CIB map are smaller than the error bars on the cross-correlation with the GASS CIB map. From Eq. (39), we can infer that the reason is the lower amount of the dust in the Planck GNILC CIB maps compared to the GASS CIB maps. These conclusions hold at 857 and $353 \mathrm{GHz}$, where the measurements are more than twice lower than what is expected from the model at $\ell=50$.

The middle panel of the Fig. 6 of Planck Collaboration Int. XLIX (2016) shows that for $545 \mathrm{GHz}$, the best-fit Planck CIB and the GNILC CIB power spectra begin to agree only after $\ell \sim 800$. Figure A.2 shows the $\ell^{3} C_{\ell}^{\mathrm{CIB} \times \phi}$ computed for the GASS CIB map and the Planck GNILC CIB map for $545 \mathrm{GHz}$ from $\ell=500$ to $\ell=1000$. Overall, although the cross-correlation with the GASS CIB map agrees better with the Maniyar et al. (2018) model than with the Planck GNILC CIB map, the GNILC CIB maps perform better at these multipoles than at multipoles $<100$ (but still systematically underestimate the cross-correlation). This shows once again an excessive cleaning of galactic dust in the Planck GNILC

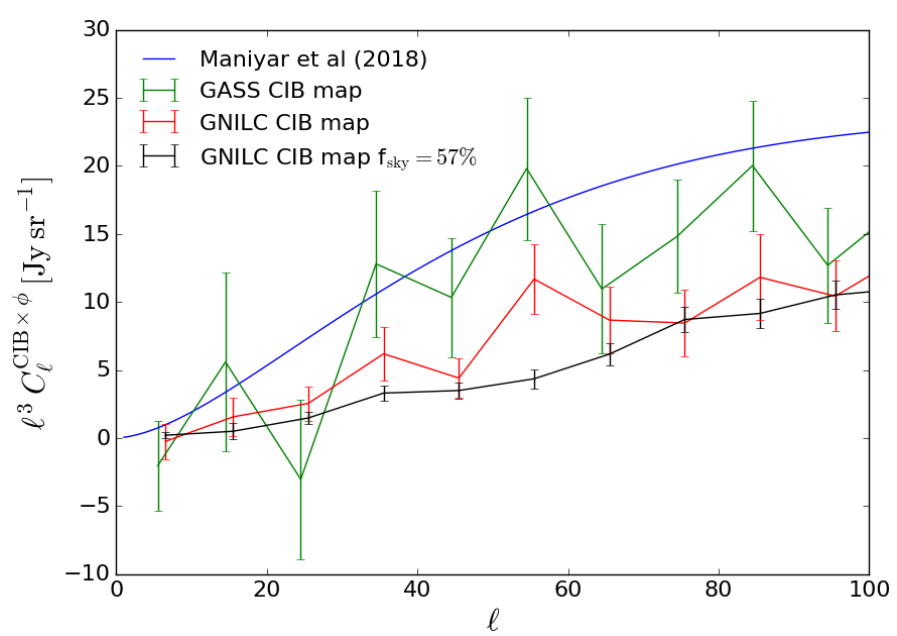

Fig. A.1. $\mathrm{CIB} \times \mathrm{CMB}$ lensing cross-power spectra for $2 \leq \ell \leq 100$ at $545 \mathrm{GHz}$. The blue curve shows the $\mathrm{CIB} \times \mathrm{CMB}$ lensing model from Maniyar et al. (2018). The green, red, and black curves show the measured cross-correlation for GASS CIB map, GASS GNILC map, and GNILC maps on $57 \%$ of the sky, respectively. The cross-correlation is consistently below the Maniyar et al. (2018) model for the GNILC CIB map. The cross-correlation obtained with the GASS CIB map agrees better with the Maniyar et al. (2018) model.

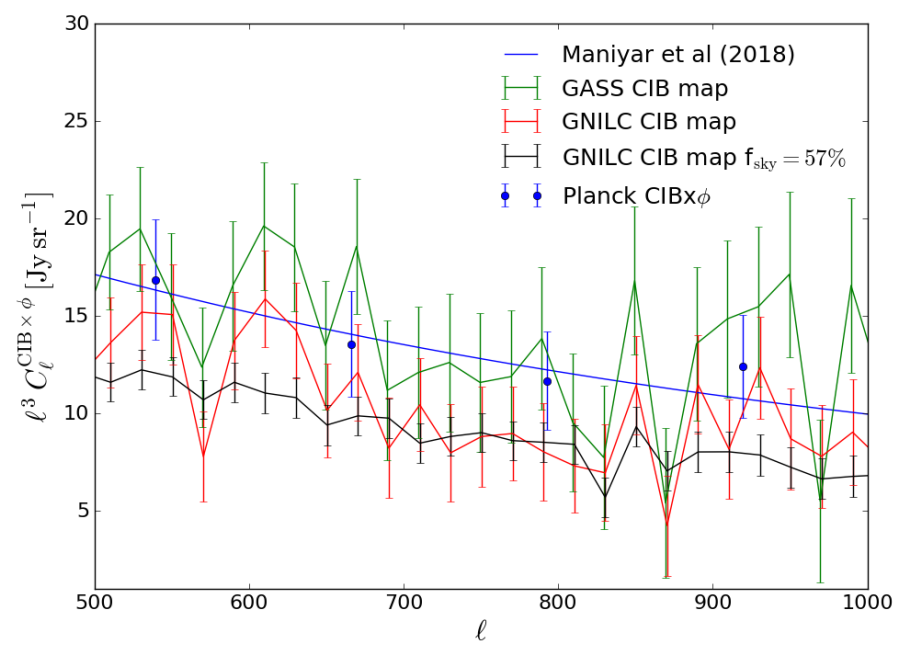

Fig. A.2. $\mathrm{CIB} \times \mathrm{CMB}$ lensing cross-power spectra for $500 \leq \ell \leq$ 1000 at $545 \mathrm{GHz}$. The blue curve shows the $\mathrm{CIB} \times \mathrm{CMB}$ lensing crosscorrelation model by Maniyar et al. (2018). The green and red curves show the measured cross-correlation in the GASS field for the CIB maps from Planck Collaboration XXX (2014) and GNILC, respectively. The black curve is the cross-correlation using GNILC CIB map on $57 \%$ of the sky. Data points in blue show the $\mathrm{CIB} \times \phi$ measurements by Planck (Planck Collaboration XVIII 2014). The cross-correlation for the GASS CIB map agrees better with the Maniyar et al. (2018) model than for the Planck GNILC CIB map.

CIB maps, which results in partial CIB removal and hence an underestimate of the CIB power. This is the reason for our decision against this CIB map in our analysis.

\footnotetext{
6 https://wiki.cosmos.esa.int/planckpla2015/index.php/ Specially_processed_maps\#2015_Lensing_map
} 